\title{
THE QUANTUM MCKAY CORRESPONDENCE FOR POLYHEDRAL SINGULARITIES
}

\author{
JIM BRYAN AND AMIN GHOLAMPOUR
}

\begin{abstract}
Let $G$ be a polyhedral group, namely a finite subgroup of $S O(3)$. Nakamura's G-Hilbert scheme provides a preferred Calabi-Yau resolution $Y$ of the polyhedral singularity $C^{3} / G$. The classical McKay correspondence describes the classical geometry of $Y$ in terms of the representation theory of $G$. In this paper we describe the quantum geometry of $Y$ in terms of $R$, an ADE root system associated to $G$. Namely, we give an explicit formula for the Gromov-Witten partition function of $Y$ as a product over the positive roots of $R$. In terms of counts of BPS states (GopakumarVafa invariants), our result can be stated as a correspondence: each positive root of $R$ corresponds to one half of a genus zero BPS state. As an application, we use the Crepant Resolution Conjecture to provide a full prediction for the orbifold Gromov-Witten invariants of $\left[\mathbb{C}^{3} / G\right]$.
\end{abstract}

\section{INTRODUCTION}

Let $G$ be a finite subgroup of $S U(3)$, and let $X$ be the quotient singularity

$$
X=\mathbb{C}^{3} / G \text {. }
$$

There is a preferred Calabi-Yau resolution

$$
\pi: Y \rightarrow X
$$

given by Nakamura's G-Hilbert scheme (Definition 6 )

$$
Y=G-\operatorname{Hilb}\left(\mathbb{C}^{3}\right) .
$$

The classical McKay correspondence describes the geometry of $Y$ in terms of the representation theory of $G$ [5, 31]. One of the original formulations 1$]$ of the correspondence is a bijection of finite sets:

\section{Classical McKay Correspondence:}

$\{$ Irreducible representations of $G\} \longleftrightarrow$ Geometric basis for $H^{*}(Y, \mathbb{Z})$.

\footnotetext{
${ }^{1}$ The modern way to formulate the correspondence is an equivalence of derived categories [5].
} 
We consider the case where $G$ is a polyhedral group, namely a finite subgroup of $S O(3) \subset S U(3)$. Such groups are classified into three families $A_{n}, D_{n}$, and $E_{n}$, given by the cyclic groups, the dihedral groups, and the symmetries of the platonic solids. In this paper we describe the quantum geometry of $Y$, namely its Gromov-Witten theory, in terms of the associated ADE root system $R$. Our main theorem provides a closed formula for the Gromov-Witten partition function in terms of the root system $R$ (Theorem 1).

This result can also be formulated in terms of the Gopakumar-Vafa invariants which are defined in terms of Gromov-Witten invariants and physically correspond to counts of BPS states. Our result provides a quantum McKay correspondence which can be stated as a natural bijection of finite sets:

\section{Quantum McKay Correspondence:}

$$
\left\{\begin{array}{c}
\text { Positive roots of } R \\
\text { other than binary roots }
\end{array}\right\} \longleftrightarrow\left\{\begin{array}{l}
\text { Contributions of } \frac{1}{2} \text { to } \\
\text { BPS state counts of } Y
\end{array}\right\}
$$

The binary roots are a distinguished subset of the simple roots and are given in Figure 1. In the above correspondence, all the BPS state counts are in genus 0 ; we show that $Y$ has no higher genus BPS states. The meaning of the above bijection is that $n_{\beta}^{0}(Y)$, the GopakumarVafa invariant which counts genus zero BPS states in the curve class $\beta$, is given by half the number of positive roots corresponding to the class $\beta$. We will explain below how the positive roots of $R$ (other than binary roots) naturally correspond to curve classes in $Y$. In general, many positive roots may correspond to the same curve class and moreover, that curve class may be reducible and/or non-reduced. Thus the above simple correspondence leads to a complicated spectrum of BPS states: they can be carried by complicated curves and have various multiplicities. As an example, we work out the $D_{5}$ case explicitly in $\S 4$.

Our method for computing the Gromov-Witten partition function of $Y$ uses a combination of localization, degeneration, and deformation techniques. There is a singular fibration $Y \rightarrow \mathbb{C}$ by (noncompact) K3 surfaces and we use localization and degeneration to relate the partition function of $Y$ to the partition function of a certain smooth $K 3$ fibration $\widehat{Y} \rightarrow \mathbb{C}$. We compute the partition function of $\widehat{Y}$ by deformation methods. The root system $R$ makes its appearance 
in the versal deformation space of the central fiber of $\widehat{Y} \rightarrow \mathbb{C}$. We give a brief overview of the proof in $\S 2.1$.

1.1. The main result. Since $G$ is a finite subgroup of $S O(3)$, it preserves the quadratic form $t=x^{2}+y^{2}+z^{2}$ and hence $G$ acts fiberwise on the family of surfaces

$$
\begin{aligned}
\mathbb{C}^{3} & \rightarrow \mathbb{C} \\
(x, y, z) & \mapsto x^{2}+y^{2}+z^{2} .
\end{aligned}
$$

Let

$$
Q_{t}=\left\{x^{2}+y^{2}+z^{2}=t\right\} \subset \mathbb{C}^{3}
$$

be the fiber over $t \in \mathbb{C}$. The central fiber is a quadric cone, which is isomorphic to the $A_{1}$ surface singularity:

$$
Q_{0} \cong \mathbb{C}^{2} /\{ \pm 1\} \text {. }
$$

Via the functorial properties of the G-Hilbert scheme, $Y$ inherits a map

$$
\epsilon: Y \rightarrow \mathbb{C}
$$

whose general fiber, $S_{t}$, is the minimal resolution of $Q_{t} / G$, and whose central fiber, $S_{0}$, is a partial resolution of

$$
Q_{0} / G \cong \mathbb{C}^{2} / \widehat{G},
$$

where $\widehat{G}$ is the binary version of $G$, namely the preimage of $G$ under the double covering $j$ :

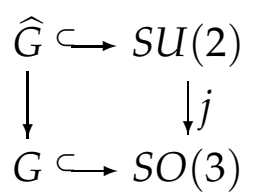

Let $\widehat{S}=\widehat{G}-\mathrm{Hilb}\left(\mathbb{C}^{2}\right)$ be the minimal resolution of the surface singularity $\mathbb{C}^{2} / \widehat{G}$

$$
\widehat{\pi}: \widehat{S} \rightarrow \mathbb{C}^{2} / \widehat{G} .
$$

The map $\widehat{\pi}$ factors through $\widehat{S} \rightarrow S_{0}$ which gives rise to a map

$$
f: \widehat{S} \rightarrow Y \text {. }
$$

See Figure 2 in $\$ 2$ for a diagram of these maps.

The map $f$ is constructed modularly by Boissiere and Sarti [4] who prove a beautiful compatibility between the McKay correspondences for $\widehat{\pi}: \widehat{S} \rightarrow \mathbb{C}^{2} / \widehat{G}$ and $\pi: Y \rightarrow \mathbb{C}^{3} / G$. The compatibility can be expressed as the commutativity of the following diagram of set maps. 
$\left\{\begin{array}{l}\text { Non-trivial irreducible } \\ \widehat{G} \text {-representations, } \widehat{\rho}\end{array}\right\} \hookrightarrow\left\{\begin{array}{l}\text { Components } \\ \text { of } \widehat{\pi}^{-1}(0), \widehat{C}_{\widehat{\rho}}\end{array}\right\}$

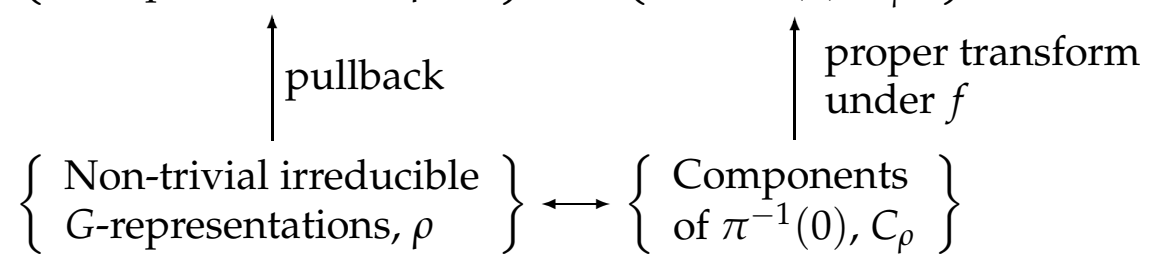

The vertical arrows are injections and horizontal arrows are the bijections from the McKay correspondences in dimensions two and three. The bijections say that the irreducible components of the exceptional fibers over 0 (which are smooth rational curves in these cases) are in natural correspondence with non-trivial irreducible representations of $\widehat{G}$ and $G$ respectively. The commutativity of the diagram says that the map $f$ contracts exactly those exceptional curves which correspond to representations of $S U(2)$ not coming from representations of $S O(3)$.

The configurations of curves in $\widehat{\pi}^{-1}(0)$ and $\pi^{-1}(0)$ are given in [4, Figures 5.1\& 5.2]. The curves $\widehat{C}_{\widehat{\rho}}$ are smooth rational curves meeting transversely with an intersection graph of ADE type. This gives rise to an ADE root system $R$ and a natural bijection between the simple roots of $R$ and the irreducible components of $\widehat{\pi}^{-1}(0)$.

$\left\{\begin{array}{l}\text { Non-trivial irreducible } \\ \widehat{G} \text {-representations, } \widehat{\rho}\end{array}\right\} \hookrightarrow\left\{\begin{array}{l}\text { Components } \\ \text { of } \widehat{\pi}^{-1}(0), \widehat{C}_{\widehat{\rho}}\end{array}\right\} \leftrightarrow\left\{\begin{array}{l}\text { Simple roots } \\ \text { of } R, e_{\widehat{\rho}}\end{array}\right\}$

For any positive root

$$
\alpha=\sum_{\widehat{\rho}} \alpha^{\widehat{\rho}} e_{\widehat{\rho}} \in R^{+}
$$

we define maps

$$
\widehat{c}: R^{+} \rightarrow H_{2}(\widehat{S}, \mathbb{Z}) \quad c: R^{+} \rightarrow H_{2}(Y, \mathbb{Z})
$$

by

$$
\widehat{c}(\alpha)=\sum_{\widehat{\rho}} \alpha \widehat{\rho}^{\widehat{C}} \widehat{C}_{\widehat{\rho}} \quad c(\alpha)=f_{*}(\widehat{c}(\alpha))
$$

Note that $\widehat{c}$ is injective but $c$ is not since $f_{*}$ contracts curves. By definition, a simple root is binary if it is in the kernal of $c$, or in other words, if the corresponding curve is contracted by $f: \widehat{S} \rightarrow Y$. See Figure 1 for a case by case description of binary roots. 


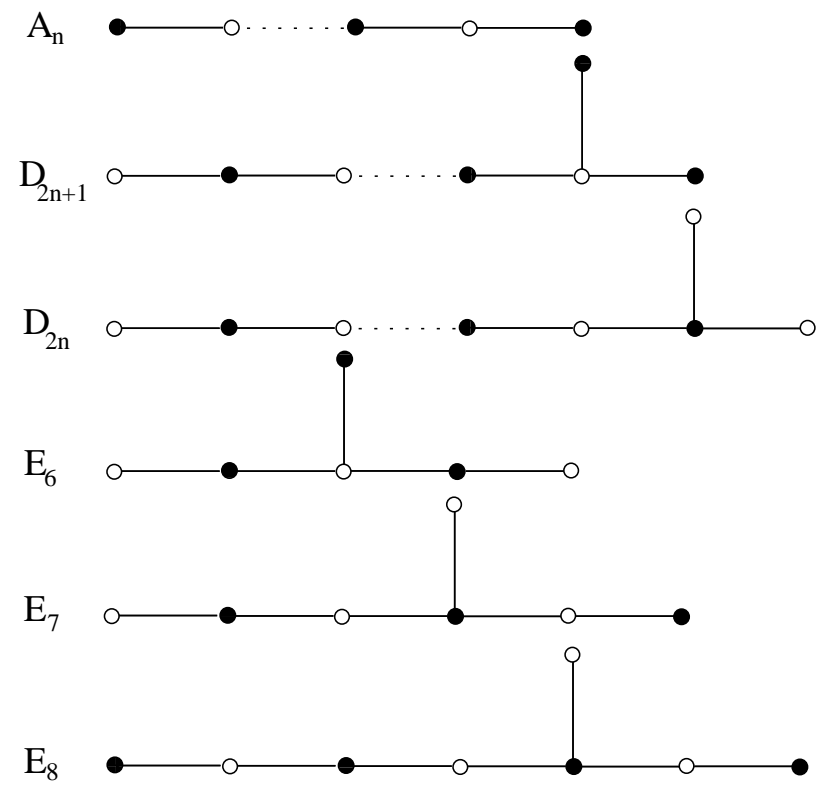

FIGURE 1. The set of binary roots of an ADE root system is the subset of the simple roots corresponding to the black nodes in the above Dynkin diagrams. The ADE Dynkin diagrams are dual to the configurations of exceptional curves in $\widehat{S}$. The map $f: \widehat{S} \rightarrow Y$ contracts exactly the curves corresponding to binary roots.

Let $N_{\beta}^{g}(Y)$ be the genus $g$ Gromov-Witten invariant of $Y$ in the class $\beta \in H_{2}(Y, \mathbb{Z})$. It is defined to be the degree of the virtual fundamental cycle on $\bar{M}_{g}(Y, \beta)$, the moduli space of genus $g$ stable maps to $Y$ in the class of $\beta$ [19, 24]. In the event that $\bar{M}_{g}(Y, \beta)$ is non-compact, as it is for some classes $\beta$, the invariant $N_{\beta}^{g}(Y)$ is defined via virtual localization [12, §2] using the natural $\mathbb{C}^{*}$ action on $Y$. The action on $Y$ is induced by the diagonal action of $\mathbb{C}^{*}$ on $\mathbb{C}^{3}$.

The Gromov-Witten invariants of non-zero degree are assembled into a generating function called the reduced Gromov-Witten partition function

$$
Z^{Y}(q, \lambda)=\exp \left(\sum_{g=0}^{\infty} \sum_{\beta \neq 0} N_{\beta}^{g}(Y) q^{\beta} \lambda^{2 g-2}\right) .
$$

The main theorem of this paper can then be written as follows. 
Theorem 1. Let $G$ be a finite subgroup of $S O(3)$. The reduced GromovWitten partition function of $Y=G-\operatorname{Hilb}\left(\mathbb{C}^{3}\right)$ is given by

$$
Z^{Y}(q, \lambda)=\prod_{\substack{\alpha \in R^{+} \\ c(\alpha) \neq 0}} \prod_{m=1}^{\infty}\left(1-q^{c(\alpha)}\left(-e^{i \lambda}\right)^{m}\right)^{m / 2}
$$

1.2. Applications. There are very few Calabi-Yau threefolds for which the Gromov-Witten partition function has been completely determined. In addition to the G-Hilbert schemes of this paper, the only other cases are toric Calabi-Yau threefolds and local curves. The partition function of a toric Calabi-Yau threefold can be computed via the topological vertex formalism [2, 27] and the partition function of a local curve is determined in [12]. Having an explicit exact formula for the Gromov-Witten partition function leads to a number of applications some of which we describe below.

1.2.1. Donaldson-Thomas and Pandharipande-Thomas theory. The Donaldson-Thomas invariants of a threefold are defined in terms of the moduli space of ideal sheaves on the threefold. It was conjectured in [29, Conj. 3] and [12, Conj. 3R] that the reduced partition function of Gromov-Witten and Donaldson-Thomas theory are equal after the change of variables $-e^{i \lambda}=Q$. Recently, a conjecturally equivalent theory using a moduli space of stable pairs has been defined by Pandharipande and Thomas [34]. Since the dependence of the genus parameter $\lambda$ in our formula for the Gromov-Witten partition function of $Y$ is explicitly through the quantity $-e^{i \lambda}$, Theorem 1 immediately yields a prediction for the reduced Donaldson-Thomas partition function of $Y$ and the Pandharipande-Thomas partition function of $Y$.

1.2.2. BPS states. The result of Theorem 1 in terms of the BPS state counts is remarkably simple. BPS state counts, defined physically by Gopakumar and Vafa [22], can be defined mathematically in terms of Gromov-Witten invariants [11, Def. 1.1]. Namely, the genus $g$, degree $\beta$ Gopakumar-Vafa invariants of $Y$, denoted by $n_{\beta}^{g}(Y)$, are defined by the formula

$$
\sum_{g=0}^{\infty} \sum_{\beta \neq 0} N_{\beta}^{g}(Y) q^{\beta} \lambda^{2 g-2}=\sum_{g=0}^{\infty} \sum_{\beta \neq 0} n_{\beta}^{g}(Y) \sum_{d>0} \frac{1}{d}\left(2 \sin \left(\frac{d \lambda}{2}\right)\right)^{2 g-2} q^{d \beta}
$$


It is well known that each genus zero BPS state $n_{\beta}^{0}$ contributes a factor of

$$
\prod_{m=1}^{\infty}\left(1-q^{\beta}\left(-e^{i \lambda}\right)^{m}\right)^{m n_{\beta}^{0}}
$$

to the partition function (e.g. [3, Proof of Thm 3.1]) and so Theorem 1 implies that BPS states of $G-\operatorname{Hilb}\left(\mathbb{C}^{3}\right)$ are given by

$$
n_{\beta}^{g}(Y)= \begin{cases}\frac{1}{2}\left|c^{-1}(\beta)\right| & g=0, \\ 0 & g>0,\end{cases}
$$

where $|\cdot|$ denotes the cardinality of a finite set. This makes precise the Quantum McKay Correspondence which we described earlier. Namely, each non-binary positive root $\alpha$ contributes $\frac{1}{2}$ to the genus 0 BPS state count in the class $\beta=c(\alpha)$. Note that the binary roots are precisely those roots $\alpha$ with $c(\alpha)=0$. The example of $D_{5}$ is worked out explicitly in $\S 4$

Remark 2. It can be directly observed that for any curve class $\beta$ in $Y,\left|c^{-1}(\beta)\right| \in\{0,1,2,4,8\}$. In particular, for some of the curve classes the BPS state count is $1 / 2$. This does not contradict the conjectural integrality of the BPS state counts of the Calabi-Yau threefolds. This is because of the non compactness of $Y$ that causes the ordinary Gromov-Witten invariant corresponding to some particular curve classes not to be a priori well defined. We use the $\mathbb{C}^{*}$ action on $Y$ to define the equivariant version of the invariants for such curve classes. The equivariant Gromov-Witten invariants agree with the ordinary Gromov-Witten invariants whenever the latter are welldefined.

1.2.3. Orbifold Gromov-Witten theory of $\left[\mathbb{C}^{3} / G\right]$. Let $\mathcal{X}$ be the orbifold associated to the singular space $\mathbb{C}^{3} / G$. In $\S 3$ we show that Theorem 1. together with classical invariants of $Y$ and the Crepant Resolution Conjecture [8, 18], give a prediction for the genus zero GromovWitten invariants of $\mathcal{X}$. To express this, we introduce some notation.

The inertia orbifold, $I \mathcal{X}$ is a union of contractible connected components, $I \mathcal{X}_{(g)}$ indexed by $(g) \in \operatorname{Conj}(G)$, the set of the conjugacy classes of $G$. Recall that the (equivariant) orbifold cohomology of $\mathcal{X}$ is by definition [14]

$$
H_{\text {orb }}^{*}(\mathcal{X})=\bigoplus_{(g) \in \operatorname{Conj}(G)} H_{C^{*}}^{*-2 \imath(g)}\left(I \mathcal{X}_{(g)}\right),
$$


where $\imath_{(g)}$ is the degree shifting number (age) of the component indexed by $(g) \in \operatorname{Conj}(G)$.

It is a consequence of $G \subset S O(3)$ that all non-trivial elements of $G$ act on $\mathbb{C}^{3}$ with age one (c.f. Lemma 24). Therefore, there exists a canonical basis $\left\{\delta_{(g)}\right\}_{(g) \in \operatorname{Conj}(G)}$, for $H_{o r b}^{*}(\mathcal{X})$ where

$$
\delta_{(e)} \in H_{\text {orb }}^{0}(\mathcal{X}) \quad \text { and } \quad \delta_{(g)} \in H_{\text {orb }}^{2}(\mathcal{X}) \text { for }(g) \neq(e) .
$$

Let $x=\left\{x_{(g)}\right\}_{(g) \in \operatorname{Conj}(G)}$ be a set of variables parameterizing this cohomology basis. For any given vector $n=\left(n_{(g)}\right)_{(g) \in \operatorname{Conj}(G)}$ of nonnegative integers, we use the following notation

$$
\delta^{n}=\prod_{(g) \in \operatorname{Conj}(G)} \delta_{(g)}^{n_{(g)}} \quad \text { and } \quad \frac{x^{n}}{n !}=\prod_{(g) \in \operatorname{Conj}(G)} \frac{x_{(g)}^{n_{(g)}}}{n_{(g)} !} .
$$

Suppose

$$
|n|=\sum_{(g) \in \operatorname{Conj}(G)} n_{(g)} .
$$

We denote the genus zero, $|n|$-point, equivariant orbifold GromovWitten invariants of $\mathcal{X}$ corresponding to the vector $n$ by $\left\langle\delta^{n}\right\rangle$ (see [14, 1] and [8, §1.4]).

Definition 3. Using the notation above, we write the genus zero orbifold Gromov-Witten potential function of $\mathcal{X}$ as

$$
F^{\mathcal{X}}(x)=\sum_{n \in \mathbb{Z}_{+}^{r}}\left\langle\delta^{n}\right\rangle \frac{x^{n}}{n !}
$$

where $r=|\operatorname{Conj}(G)|$. For simplicity, we set the variable $x_{(e)}$ to zero (see Remark 23).

In $\S 3$, we establish the following prediction for $F^{\mathcal{X}}(x)$. The reader can find a similar prediction for the orbifold Gromov-Witten invariants of $\left[\mathbb{C}^{2} / \widehat{G}\right]$ given in [6, Conjecture 11].

Conjecture 4. Let $\mathrm{G}$ be a finite subgroup of $\mathrm{SO}(3)$ and let $\mathbf{h}(s)$ be a series defined by

$$
\mathbf{h}^{\prime \prime \prime}(s)=\frac{1}{2} \tan \left(-\frac{s}{2}\right) .
$$

Let

$$
X_{\rho}=\frac{1}{|G|}\left(2 \pi \operatorname{dim}(\rho)+\sum_{g \in G} \sqrt{3-\chi_{V}(g)} \chi_{\rho}(g) x_{(g)}\right)
$$


where $\chi_{\rho}(g)$ is the character of the G-representation $\rho$ evaluated on $g$ and $V$ is the three dimensional representation induced by the embedding $G \subset$ $S O(3) \subset S U(3)$.

Then the genus zero orbifold Gromov-Witten potential function of $\mathcal{X}=$ $\left[\mathbb{C}^{3} / G\right]$ is given by

$$
F^{\mathcal{X}}(x)=\frac{1}{2} \sum_{\alpha \in R^{+}} \mathbf{h}\left(\pi+\sum_{\rho} \alpha^{\rho} \mathrm{X}_{\rho}\right)
$$

where the second sum is over non-trivial irreducible G-representations $\rho$ and $\alpha^{\rho}$ is the coefficient of $e_{\rho}$ (the simple root corresponding to $\rho$ ) in the positive root $\alpha$.

Note that terms of order less than three in $\mathbf{h}(s)$, and consequently in $F^{\mathcal{X}}(x)$, are not defined. This reflects the fact that the invariants with fewer than three insertions are also not defined. The orbifold Gromov-Witten invariants of $\left[\mathbb{C}^{3} / G\right]$ may be expressed in terms of G-Hurwitz-Hodge integrals. Hurwitz-Hodge integrals have been the topic of extensive recent research [7, 9, 13, 15, 16, 35, 39].

In $\$ 3$ we prove the following proposition.

Proposition 5. If Conjecture 4 is true, then the genus zero crepant resolution conjecture holds for $Y \rightarrow \mathbb{C}^{3} / G$.

Conjecture 4 has been proved for $G$ equal to $\mathbb{Z}_{2} \times \mathbb{Z}_{2}$ or $A_{4}$ in [7] (which correspond to $D_{4}$ and $E_{6}$ in the ADE classification), and for $\mathbb{Z}_{n+1}$ in [16] (which corresponds to $A_{n}$ in the ADE classification). Zhou has recently proved a higher genus analog of Conjecture 4 in the $A_{n}$ case [38].

\section{Proof of Theorem 1 .}

2.1. Overview. To compute the Gromov-Witten partition function of $Y$, we construct and study four families of (non-compact) K3 surfaces:

$$
\begin{array}{ll}
\epsilon: Y \rightarrow \mathbb{C} & \widehat{\epsilon}: \widehat{Y} \rightarrow \mathbb{C} \\
\bar{\epsilon}: \bar{Y} \rightarrow \mathbb{P}^{1} & \widehat{\bar{\epsilon}}: \widehat{\bar{Y}} \rightarrow \mathbb{P}^{1}
\end{array}
$$

We obtain the smooth family $\widehat{\epsilon}$ from $\epsilon$ by taking the double cover of $Y$ branched along the (singular) central fiber and then taking a small resolution of the resulting conifold singularities. The family $\bar{\epsilon}$ extends the family $\epsilon$ to the compact base $\mathbb{P}^{1}$. The family $\widehat{\bar{\epsilon}}$ extends the family $\widehat{\epsilon}$ to the compact base $\mathbb{P}^{1}$. 
We study $Z^{Y}(q, \lambda)$ and $Z^{\widehat{Y}}(\widehat{q}, \lambda)$, the reduced Gromov-Witten partition functions of $Y$ and $\widehat{Y}$ respectively. We also study $Z_{f}^{\bar{Y}}(q, \lambda)$ and $Z_{f}^{\hat{\bar{Y}}}(\widehat{q}, \lambda)$, the fiber class reduced Gromov-Witten partition functions of $\bar{Y}$ and $\widehat{\bar{Y}}$ respectively.

We denote the set of non-trivial irreducible representations of $G$ and $\widehat{G}$ by $\operatorname{Irr}^{*}(G)$ and $\operatorname{Irr}^{*}(\widehat{G})$, respectively. Using the identifications in Lemma 11, the quantum variables of $Y$ and the fiber class quantum variables of $\bar{Y}$ are given by $\left\{q_{\rho}\right\}_{\rho \in \operatorname{Irr}^{*}(G)}$ whereas the quantum variables of $\widehat{Y}$ and the fiber class quantum variables of $\widehat{\bar{Y}}$ are given by $\left\{\widehat{q}_{\widehat{\rho}}\right\}_{\widehat{\rho} \in \operatorname{Irr}^{*}(\widehat{G})}$.

There is a specialization of the variables given by

$$
\widehat{q} \widehat{\rho}= \begin{cases}q_{\rho} & \text { if } \widehat{\rho} \text { pulls back from } \rho \in \operatorname{Irr}^{*}(G) \\ 1 & \text { if } \widehat{\rho} \text { is not the pullback of a } G \text { representation. }\end{cases}
$$

We will prove the following four equalities

$$
\begin{aligned}
& Z^{Y}(q, \lambda)^{2}=Z_{f}^{\bar{Y}}(q, \lambda) \\
& Z_{f}^{\bar{Y}}(q, \lambda)^{2}=\frac{Z_{f}^{\hat{\bar{Y}}}(\widehat{q}, \lambda)}{\left.Z_{f}^{\hat{\bar{Y}}}(\widehat{q}, \lambda)\right|_{q=0}} \\
& Z_{f}^{\hat{\bar{Y}}}(\widehat{q}, \lambda)=Z^{\widehat{Y}}(\widehat{q}, \lambda)^{2} \\
& Z^{\widehat{Y}}(\widehat{q}, \lambda)=\prod_{\alpha \in R^{+}} \prod_{m=1}^{\infty}\left(1-\widehat{q} \widehat{c}(\alpha)\left(-e^{i \lambda}\right)^{m}\right)^{m}
\end{aligned}
$$

where the $\widehat{q} \mapsto q$ specialization is implicit in equation (2). The main theorem follows immediately from these four equations.

In $\$ 2.2$ we discuss the geometry of the G-Hilbert scheme $Y$ and we construct the four families. In Proposition 12 we prove (4) using a deformation argument. In Lemma 14 we prove (3) and (1) using a localization argument. In Proposition 15 we prove (2) using a degeneration argument and the Li-Ruan formula for the Gromov-Witten invariants of a conifold transition [26].

2.2. Geometry of $Y=G-\operatorname{Hilb}\left(\mathbb{C}^{3}\right)$ and the families $\epsilon, \widehat{\epsilon}, \bar{\epsilon}, \widehat{\bar{\epsilon}}$.

We start by recalling the definition of $Y$, our main object of study. 
Definition 6. Let $Y=G-H i l b\left(\mathbb{C}^{3}\right)$ be Nakamura's G-Hilbert scheme [33], namely the subscheme of $\mathrm{Hilb}^{|G|}\left(\mathbb{C}^{3}\right)$ parameterizing closed Ginvariant subschemes $Z \subset \mathbb{C}^{3}$ such that $H^{0}\left(\mathcal{O}_{Z}\right)$ is isomorphic to the regular representation of $G$. Here $\mathrm{Hilb}^{|G|}\left(\mathbb{C}^{3}\right)$ denotes the Hilbert scheme of length $|G|$ subschemes of $\mathbb{C}^{3}$.

Consider the Hilbert-Chow morphism from $\operatorname{Hilb}^{|G|}\left(\mathbb{C}^{3}\right)$ to the $|G|$ th symmetric product of $\mathbb{C}^{3}$

$$
h: \operatorname{Hilb}^{|G|}\left(\mathbb{C}^{3}\right) \rightarrow \operatorname{Sym}^{|G|}\left(\mathbb{C}^{3}\right)
$$

that sends a closed subscheme $Z \subset \mathbb{C}^{3}$ to the zero cycle $[Z]$ associated to it. The morphism $h$ is projective and it restricts to a projective morphism

$$
\pi: Y \rightarrow \mathbb{C}^{3} / G
$$

Bridgeland, King, and Reid prove that $Y$ is smooth, reduced and irreducible variety, and that $\pi$ is a crepant resolution [5, Theorem 1.2].

Remark 7. More generally, one can define the $H$-Hilbert scheme $H$-Hilb $(M)$, where $M$ is any smooth $n$-dimensional quasi-projective variety, and $H$ is a finite group of automorphisms of $M$, such that the canonical bundle of $M$ is a locally trivial $H$-sheaf [33, 36, 5].

(i) $H$-Hilb $(M)$ represents the moduli functor of $H$-clusters [4, §3].

(ii) If $n \leq 3$ then $H$-Hilb $(M) \rightarrow M / G$ is a crepant resolution [5, Theorem 1.2].

Recall that $\epsilon: Y \rightarrow \mathbb{C}$ is the composition of the map $\pi: Y \rightarrow \mathbb{C}^{3} / G$ with the map $\mathbb{C}^{3} / G \rightarrow \mathbb{C}$ given by $(x, y, z) \mapsto x^{2}+y^{2}+z^{2}$. We analyze the fibers $S_{t}=\epsilon^{-1}(t)$. The relevant morphisms are shown in the diagram in Figure 2 .

By [4, Theorem 1.1],

$$
\pi: Y \rightarrow \mathbb{C}^{3} / G
$$

restricts to a partial resolution,

$$
\pi_{0}: S_{0} \rightarrow Q_{0} / G,
$$

whose exceptional locus coincides with $\pi^{-1}(0)$.

Let $\widehat{S}=\widehat{G}-\mathrm{Hilb}\left(\mathbb{C}^{2}\right)$. Boissiere and Sarti [4, Theorems 1.1\& 8.1] relate the geometry of $\widehat{S}$ and $Y$ by constructing a morphism

$$
f: \widehat{S} \rightarrow Y
$$




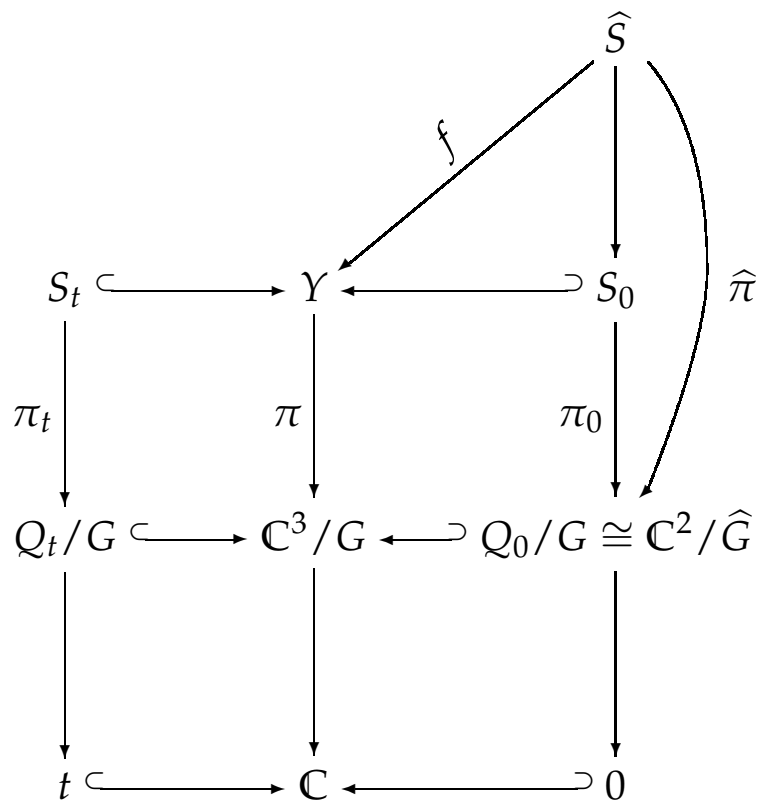

FIGURE 2. The family of surfaces $\epsilon: Y \rightarrow \mathbb{C}$ and related morphisms.

and proving it factors through $S_{0} \subset Y$ as the minimal resolution. They prove it maps $\widehat{C}_{\rho}$ isomorphically to $C_{\rho}$ if $\rho \in \operatorname{Irr}^{*}(G)$, and it contracts $\widehat{C}_{\rho}$ if $\rho \in \operatorname{Irr}^{*}(\widehat{G}) \backslash \operatorname{Irr}^{*}(G)$. By the functoriality of the $G$ Hilbert scheme (see Remark 7 (i)), for each $t \neq 0$

$$
S_{t} \cong G-\operatorname{Hilb}\left(Q_{t}\right),
$$

and by Remark $7($ ii), $\pi$ restricts to the minimal resolution

$$
\pi_{t}: S_{t} \rightarrow Q_{t} / G \text {. }
$$

Remark 8. The action of $s \in \mathbb{C}^{*}$ on $\mathbb{C}^{3}$ is given by

$$
(x, y, z) \mapsto(s x, s y, s z) \text {. }
$$

Thus in order to make the map $t=x^{2}+y^{2}+z^{2}$ equivariant, the action of $s$ on the base of the family $\epsilon$ is given by

$$
t \mapsto s^{2} t .
$$

The family $\epsilon: Y \rightarrow \mathbb{C}$ has a singular central fiber $S_{0}$. We construct a related smooth family $\widehat{\epsilon}$ as follows. Let

$$
\widehat{Y}_{\text {sing }} \rightarrow \mathbb{C}
$$


be the double cover of $Y$ branched over the central fiber $S_{0}$. The singularities of $S_{0}$ are obtained by contracting -2 curves in $\widehat{S}$, which are disjoint by [4, Figures 5.1\&5.2] (c.f. Figure 1). Hence the singularities of $S_{0}$ are isolated rational double point singularities of type $A_{1}$. Consequently, $\widehat{Y}_{\text {sing }}$ has conifold singularities at the singular points of $S_{0}$, and it is smooth away from these points. Let $\widehat{Y} \rightarrow \widehat{Y}_{\text {sing }}$ be a small resolution of the conifold singularities. Thus we obtain a $\mathbb{C}^{*}$ equivariant family

$$
\widehat{\epsilon}: \widehat{Y} \rightarrow \mathbb{C}
$$

that makes the following diagram of $\mathbb{C}^{*}$-equivariant maps commute.

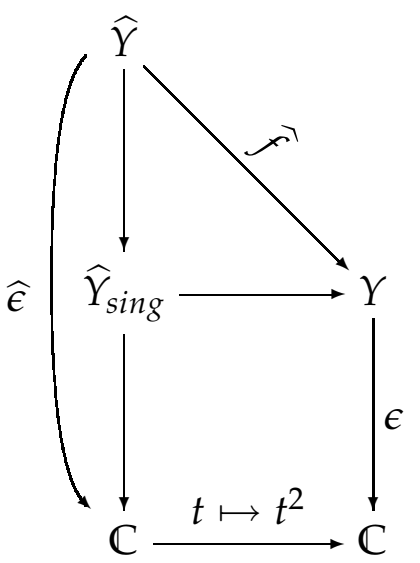

By the uniqueness of the minimal resolution in dimension two, the central fiber $\widehat{\epsilon}^{-1}(0)$ is isomorphic to $\widehat{S}$, the minimal resolution of $\mathbb{C}^{2} / \widehat{G}$. Hence, $\widehat{\epsilon}$ is a $K 3$ fibration with all the fibers being smooth, and $\left\{\widehat{C}_{\widehat{\rho}}\right\}_{\widehat{\rho} \in \operatorname{Irr}^{*}(\widehat{G})}$ forms a basis for

$$
H_{2}(\widehat{Y}, \mathbb{Z}) \cong H_{2}(\widehat{S}, \mathbb{Z})
$$

(see Lemma 11).

Remark 9. Note that because the base of the family $\widehat{\epsilon}$ double covers the base of $\epsilon$, the $\mathbb{C}^{*}$-action has weight one on the base of $\widehat{\epsilon}$.

We construct $\bar{\epsilon}$ and $\widehat{\bar{\epsilon}}$ by compactifying the base of $\epsilon$ and $\widehat{\epsilon}$ as follows. Let

$$
W=\mathbb{C}^{3} \bigcup_{\mathbb{C}^{3} \backslash Q_{0}} \mathbb{C}^{3}
$$

where the two copies of $\mathbb{C}^{3}$ are glued along $\mathbb{C}^{3} \backslash Q_{0}$ via the map

$$
(x, y, z) \mapsto\left(\frac{x}{x^{2}+y^{2}+z^{2}}, \frac{y}{x^{2}+y^{2}+z^{2}}, \frac{z}{x^{2}+y^{2}+z^{2}}\right) .
$$


The two copies of the map $\varepsilon: \mathbb{C}^{3} \rightarrow \mathbb{C}$ given by $(x, y, z) \mapsto x^{2}+y^{2}+$ $z^{2}$ patch together to give the map

$$
\bar{\varepsilon}: W \rightarrow \mathbb{P}^{1} \text {. }
$$

The actions of $\mathbb{C}^{*}$ and $G$ extends naturally to $\bar{\varepsilon}$. We define

$$
\bar{Y}=G-\operatorname{Hilb}(W) \text {. }
$$

This construction gives rise to the $\mathbb{C}^{*}$-equivariant family

$$
\bar{\epsilon}: \bar{Y} \rightarrow \mathbb{P}^{1} \text {. }
$$

Restricted to each of the $\mathbb{C}^{*}$-invariant affine patches of $\mathbb{P}^{1}$, the family is isomorphic to $\epsilon$, but with opposite $\mathbb{C}^{*}$ weights.

We can easily extend the construction of $\widehat{\epsilon}$ to the compactified base as follows.

Let

$$
\widehat{\bar{Y}}_{\text {sing }} \rightarrow \mathbb{P}^{1}
$$

be the double cover of the family $\bar{\epsilon}$ branched over the the fibers $\bar{\epsilon}^{-1}(0)$ and $\bar{\epsilon}^{-1}(\infty)$. Let

$$
\widehat{\bar{Y}} \rightarrow \widehat{\bar{Y}}_{\text {sing }}
$$

be a small resolution of the conifold singularities (chosen as stated in Remark 10 below). One obtains the family

$$
\widehat{\bar{\epsilon}}: \widehat{\bar{Y}} \rightarrow \mathbb{P}^{1}
$$

of smooth non-compact $K 3$ surfaces that make the following diagram of $\mathbb{C}^{*}$-equivariant maps commute.



Remark 10. Care must be taken in the choice of the conifold resolution to ensure that the resulting space $\widehat{\bar{Y}}$ is quasi-projective (otherwise the Gromov-Witten cannot be defined). This can be achieved by matching the resolution obtained by constructing $\widehat{\bar{Y}}$ by gluing two 
copies of $\widehat{Y}$ along $\widehat{Y}-\widehat{S}=\widehat{\epsilon}^{-1}\left(\mathbb{C}^{*}\right)$ via the involution of $\widehat{Y}-\widehat{S}$ induced by the map (5).

We define the fiberwise homology groups $H_{2}(\bar{Y}, \mathbb{Z})_{f}, H_{2}(\widehat{\bar{Y}}, \mathbb{Z})_{f}$, and $H_{2}\left(\hat{\bar{Y}}_{\text {sing }}, \mathbb{Z}\right)_{f}$ to be the kernels of the maps $\bar{\epsilon}_{*}, \widehat{\bar{\epsilon}}_{*}$, and $\left(\widehat{\bar{\epsilon}}_{\text {sing }}\right)_{*}$ respectively.

Lemma 11. The inclusions of $S_{0}$ as the fiber over 0 and over $\infty$ in $Y, \bar{Y}$, $\widehat{\bar{Y}}_{\text {sing, }}$ and $\widehat{Y}_{\text {sing }}$ induce isomorphisms in homology (taken with $\mathbb{Z}$ coefficients):

$$
H_{2}\left(S_{0}\right) \cong H_{2}(Y) \cong H_{2}\left(\widehat{Y}_{\text {sing }}\right) \cong H_{2}(\bar{Y})_{f} \cong H_{2}\left(\widehat{\bar{Y}}_{\text {sing }}\right)_{f}
$$

Moreover, the isomorphisms induced by the inclusions of the fiber over 0 and the fiber over $\infty$ are the same isomorphism. These groups all have a canonical basis given by $\left\{C_{\rho}\right\}$, the components of $\pi^{-1}(0)$, which are labelled by $\rho \in \operatorname{Irr}^{*}(G)$.

The inclusions of $\widehat{S}$ as the fiber over 0 and over $\infty$ in $\widehat{\bar{Y}}$ and $\widehat{Y}$ induce isomorphisms on homology (taken with $\mathbb{Z}$ coefficients):

$$
H_{2}(\widehat{S}) \cong H_{2}(\widehat{Y}) \cong H_{2}(\widehat{\bar{Y}})_{f}
$$

Moreover, the isomorphisms induced by the inclusions of the fiber over 0 and the fiber over $\infty$ are the same isomorphism. These groups all have a canonical basis given by $\left\{\widehat{C}_{\hat{\rho}}\right\}$, the components of $\widehat{\pi}^{-1}(0)$ which are labelled by and $\widehat{\rho} \in \operatorname{Irr}^{*}(\widehat{G})$.

PROOF: Using a triangulation of the pair $\left(Y, \pi^{-1}(0)\right)[28,23]$ and the $\mathbb{C}^{*}$ action, one can easily construct a retract of $Y$ onto $\pi^{-1}(0)$. It is well known that $S_{0}$ has a deformation retract onto $\pi^{-1}(0)$ [20]. Thus the inclusions

$$
\pi^{-1}(0) \subset S_{0} \subset Y
$$

induce isomorphisms in homology. Similar arguments apply for $\pi^{-1}(0) \subset S_{0} \subset \widehat{Y}_{\text {sing }}$ and $\widehat{\pi}^{-1}(0) \subset \widehat{S} \subset \widehat{Y}$.

To show that $H_{2}\left(S_{0}\right) \cong H_{2}(\bar{Y})_{f}$, we will use Mayer-Vietoris for the covering $\bar{Y}=Y \cup Y, Y \cap Y=Y-S_{0}$ and the compatible covering of 
$\mathbb{P}^{1}$ to obtain the following diagram

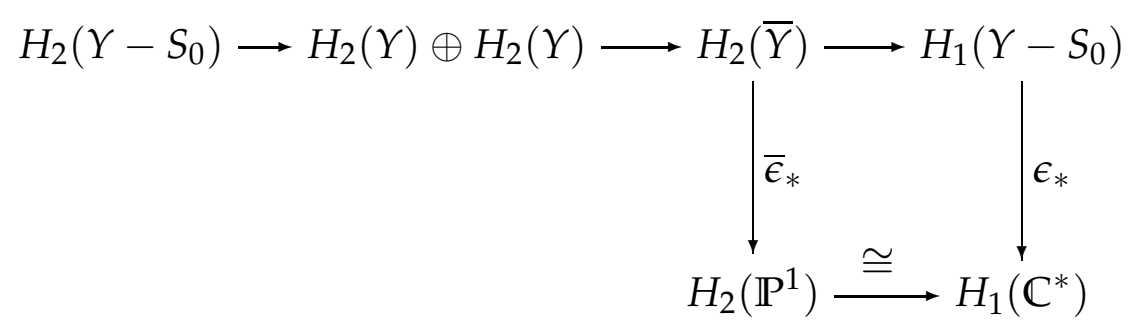

The Leray spectral sequence for the fibration

$$
\epsilon: Y-S_{0} \rightarrow \mathbb{C}^{*}
$$

degenerates at the $E_{p q}^{2}$ term and gives rise to isomorphisms:

$$
\begin{aligned}
\epsilon_{*}: H_{1}\left(Y-S_{0}\right) & \rightarrow H_{1}\left(\mathbb{C}^{*}\right) \\
i_{*}: H_{2}\left(\epsilon^{-1}(1)\right) & \rightarrow H_{2}\left(Y-S_{0}\right) .
\end{aligned}
$$

Using the above isomorphisms, the commutativity of the above diagram, and exactness, we get

$$
\begin{aligned}
H_{2}(\bar{Y})_{f} & =\operatorname{Ker}\left(H_{2}(\bar{Y}) \rightarrow H_{2}\left(\mathbb{P}^{1}\right)\right) \\
& =\operatorname{Ker}\left(H_{2}(\bar{Y}) \rightarrow H_{1}\left(Y-S_{0}\right)\right) \\
& =\operatorname{Coker}\left(H_{2}\left(Y-S_{0}\right) \rightarrow H_{2}(Y) \oplus H_{2}(Y)\right) \\
& =\operatorname{Coker}\left(H_{2}\left(\epsilon^{-1}(1)\right) \rightarrow H_{2}(Y) \oplus H_{2}(Y)\right) .
\end{aligned}
$$

By construction, the involution on $Y-S_{0}$ used to glue $Y$ to $Y$ is the identity on $\epsilon^{-1}(1)$ so the map of $H_{2}\left(\epsilon^{-1}(1)\right)$ to $H_{2}(Y) \oplus H_{2}(Y)$ lies in the diagonal. Consequently, the two maps $\mathrm{H}_{2}\left(S_{0}\right) \rightarrow \mathrm{H}_{2}(\bar{Y})_{f}$ induced by the inclusions of the fibers over 0 and $\infty$ are the same, as asserted by the Lemma. The fact that they are isomorphisms follows from the surjectivity of $H_{2}\left(\epsilon^{-1}(1)\right) \rightarrow H_{2}(Y)$.

The arguments for $H_{2}\left(S_{0}\right) \cong H_{2}\left(\widehat{\bar{Y}}_{\text {sing }}\right)_{f}$ and $H_{2}(\widehat{S}) \cong H_{2}(\widehat{\bar{Y}})_{f}$ are similar.

\subsection{The Gromov-Witten partition function of $\widehat{Y}$.}

Proposition 12. Let

$$
\widehat{c}: R^{+} \rightarrow H_{2}(\widehat{S}, \mathbb{Z}) \cong H_{2}(\widehat{Y}, \mathbb{Z})
$$

be defined as in $\$ 1$. Then the reduced Gromov-Witten partition function of $\widehat{Y}$ is given by

$$
Z^{\widehat{Y}}(\widehat{q}, \lambda)=\prod_{\alpha \in R^{+}} \prod_{m=1}^{\infty}\left(1-\widehat{q}^{\widehat{c}(\alpha)}\left(-e^{i \lambda}\right)^{m}\right)^{m} .
$$


PROOF: The proof is based on the deformation invariance of the Gromov-Witten invariants. See [10] and [6] for a similar argument.

Since $\widehat{Y}$ is smooth, $\widehat{\epsilon}$ above defines a flat family of $\mathbb{C}^{*}$-equivariant deformations of $\widehat{S}$. Thus it induces a classifying morphism

$$
\mu: \mathbb{C} \rightarrow \operatorname{Def}(\widehat{S}),
$$

where $\operatorname{Def}(\widehat{S})$ is the versal space of $\mathbb{C}^{*}$-equivariant deformations of $\widehat{S}$. $\operatorname{Def}(\widehat{S})$ is naturally identified with the complexified root space associated to the root system $R$ (see [25]). There is a bijection between the set of positive roots $R^{+}$and the irreducible components of the discriminant locus in $\operatorname{Def}(\widehat{S})$. Each irreducible component is a hyperplane in $\operatorname{Def}(\widehat{S})$ perpendicular to the corresponding positive root. A generic point of $\operatorname{Def}(\widehat{S})$ corresponds to an affine surface (having no compact curves), whereas a generic point in the component of the discriminant locus perpendicular to $\alpha \in R^{+}$, corresponds to a smooth surface with only one smooth rational curve with selfintersection -2 representing the homology class $\widehat{c}(\alpha)$ (see [25, Theorem 1] or [10, Proposition 2.2]).

By construction, $\mu$ is a non-constant $\mathbb{C}^{*}$-equivariant map from $\mathbb{C}$ to the vector space $\operatorname{Def}(\widehat{S})$.

Since $\mathbb{C}^{*}$ acts on both the source and target with weight one (see Remark 9 and [25, Theorem 1]), $\mu(t)$ must be homogeneous of degree 1. Namely $\mu$ is a linear map, and hence its image is a line passing through the origin.

Note that because $\epsilon: Y \rightarrow \mathbb{C}$ has compact curves in each fiber, the family $\widehat{\epsilon}: \widehat{Y} \rightarrow \mathbb{C}$ has compact curves in each fiber. Thus the image of $\mu$ is contained in the discriminant locus.

Let

$$
\mu_{1}: \mathbb{C} \rightarrow \operatorname{Def}(\widehat{S})
$$

be a linear map whose image is a generic line passing through the origin. Let $\widehat{Y}_{1}$ be the total space of the family induced by $\mu_{1}$. Clearly, $\widehat{Y}_{1}$ is obtained from $\widehat{Y}$ by a $\mathbb{C}^{*}$-equivariant deformation. Since the equivariant Gromov-Witten invariants are invariant under equivariant deformation we have

$$
Z^{\widehat{Y}_{1}}(\widehat{q}, \lambda)=Z^{\widehat{Y}}(\widehat{q}, \lambda)
$$

In contrast to $\mu$, the image of $\mu_{1}$ meets the discriminant locus only at the origin, hence all the curve classes of $\widehat{Y}_{1}$ are represented only by the curves supported on the central fiber of the family $\widehat{Y}_{1} \rightarrow \mathbb{C}$. Thus, for any $\beta \neq 0, N_{\beta}^{g}\left(\widehat{Y}_{1}\right)$ (and hence $Z^{\widehat{Y}_{1}}(\widehat{q}, \lambda)$ ) has a well defined 
non-equivariant limit. Consequently, we can compute the invariants of $\widehat{Y}_{1}$ (and hence of $\widehat{Y}$ ) by making a non-equivariant deformation of $\widehat{Y}_{1}$ to $\widehat{Y}_{2}$. Let

$$
\mu_{2}: \mathbb{C} \rightarrow \operatorname{Def}(\widehat{S}),
$$

be a generic affine linear map whose image is an affine line meeting the discriminant locus transversely. Let $\widehat{Y}_{2}$ be the total space of the family induced by $\mu_{2}$. Since the Gromov-Witten invariants are invariant under deformations we have

$$
Z^{\widehat{Y}_{2}}(\widehat{q}, \lambda)=Z^{\widehat{\Upsilon}_{1}}(\widehat{q}, \lambda) .
$$

By the description of the discriminant locus given above it is evident that the image of $\mu_{2}$ meets transversally each component of the discriminant locus once at a generic point. This implies that the threefold $\widehat{Y}_{2}$ contains a unique isolated smooth rational curve with normal bundle isomorphic to

$$
\mathcal{O}_{\mathbb{P}^{1}}(-1) \oplus \mathcal{O}_{\mathbb{P}^{1}}(-1)
$$

in the curve class corresponding to each element of $R^{+}$(see [6, Lemma 2]).

The contribution to the Gromov-Witten partition function of an isolated $(-1,-1)$ curve in a class $\beta$ is well known (e.g. the proof of Theorem 3.1 in [3]). It is given by a factor of

$$
\prod_{m=1}^{\infty}\left(1-q^{\beta}\left(-e^{i \lambda}\right)^{m}\right)^{m}
$$

and thus

$$
Z^{\widehat{Y}_{2}}(\widehat{q}, \lambda)=\prod_{\alpha \in R^{+}} \prod_{m=1}^{\infty}\left(1-\widehat{q}^{\widehat{c}(\alpha)}\left(-e^{i \lambda}\right)^{m}\right)^{m} .
$$

The proposition follows.

Remark 13. The image of $\mu$ in $\operatorname{Def}(\widehat{S})$ defines a line in complex root space and it is natural to ask which specific line it is. The line is not quite unique-it depends on the choice of the small resolution $\widehat{Y} \rightarrow \widehat{Y}_{\text {sing. }}$. We have found that there is a choice of resolution such that the line is generated by the vector

$$
\sum_{\widehat{\rho} \in \operatorname{Irr}^{*}(\widehat{G}) \backslash \operatorname{Irr}^{*}(G)} \operatorname{dim}(\widehat{\rho}) e_{\widehat{\rho}} .
$$

The lines corresponding to other choices of the resolution are obtained from the above line by the action of $W_{0} \subset W$, the subgroup of the Weyl group generated by reflections about $e_{\widehat{\rho}}$, where $\widehat{\rho} \in \operatorname{Irr}(\widehat{G}) \backslash \operatorname{Irr}(G)$. The specific form of the generating vector given above was not needed 
in the proof of Proposition 12, but it does provide a concrete description of $\widehat{Y}$ and hence $Y$ via the explicit description of the universal family over $\operatorname{Def}(\widehat{S})$ given in [25].

\subsection{Relating the Gromov-Witten theories of $Y, \widehat{Y}, \bar{Y}$, and $\widehat{\bar{Y}}$.}

Lemma 14. The partition functions of $Y$ and $\widehat{Y}$ are related to the fiber class partition functions of $\bar{Y}$ and $\widehat{\bar{Y}}$ by the formulas

$$
\begin{aligned}
& Z^{Y}(q, \lambda)^{2}=Z_{f}^{\bar{Y}}(q, \lambda), \\
& Z^{\widehat{Y}}(\widehat{q}, \lambda)^{2}=Z_{f}^{\hat{\bar{Y}}}(\widehat{q}, \lambda) .
\end{aligned}
$$

PROOF: The $\mathbb{C}^{*}$-fixed part of the moduli space of stable maps to $\bar{Y}$ representing fiber classes has two isomorphic components, corresponding to maps with images in either of $\bar{\epsilon}^{-1}(0)$ or $\bar{\epsilon}^{-1}(\infty)$. Each component is isomorphic to the $\mathbb{C}^{*}$-fixed part of the moduli space of stable maps to $Y$. Since the virtual dimension of the moduli space of stable maps to $\bar{Y}$ representing $\beta \in H_{2}(\bar{Y}, \mathbb{Z})_{f}$ is zero, the corresponding Gromov-Witten invariant $N_{\beta}^{g}(\bar{Y})$ is independent of the weight of the $\mathbb{C}^{*}$ action. We using the isomorphisms in Lemma 11 to identify the second homology groups of $\bar{\epsilon}^{-1}(0)$ and $\bar{\epsilon}^{-1}(\infty)$ with $H_{2}(\bar{Y}, \mathbb{Z})_{f}$. Then by localization, we have $N_{\beta}^{g}(\bar{Y})=2 N_{\beta}^{g}(Y)$. The same argument yields $N_{\beta}^{g}(\widehat{\bar{Y}})=2 N_{\beta}^{g}(\widehat{Y})$. The lemma follows.

Finally we relate the Gromov-Witten theory of $\widehat{\bar{Y}}$ to the GromovWitten theory of $\bar{Y}$. This is given in the following proposition:

Proposition 15. The reduced Gromov-Witten partition function of $\widehat{\bar{Y}}$ and $\bar{Y}$ satisfy the relation

$$
\frac{Z_{f}^{\hat{\bar{Y}}}(\widehat{q}, \lambda)}{\left.Z_{f}^{\hat{\bar{Y}}}(\widehat{q}, \lambda)\right|_{q=0}}=Z_{f}^{\bar{Y}}(q, \lambda)^{2}
$$

after the specialization

$$
\widehat{q}_{\widehat{\rho}}= \begin{cases}q_{\rho} & \text { if } \widehat{\rho} \text { pulls back from } \rho \in \operatorname{Irr}^{*}(G) \\ 1 & \text { if } \widehat{\rho} \text { is not the pullback of a G representation. }\end{cases}
$$

PROOF: Since $\bar{Y}$ is a family over a compact base, and each fiber of $\bar{\epsilon}$ is a non-compact surface but with a finite number of compact curves, the moduli space of stable maps to $\bar{Y}$ representing a fiber 
class is compact. Thus, $Z_{f}^{\bar{Y}}(q, \lambda)$ has a non-equivariant limit. All the elements of $H_{2}(\widehat{\bar{Y}}, \mathbb{Z})_{f}$ are Calabi-Yau classes, and by the same argument, the corresponding Gromov-Witten partition function, $Z_{f} \widehat{\bar{Y}}(\widehat{q}, \lambda)$, has a non equivariant limit.

Let

$$
\widehat{\bar{\epsilon}}_{\text {gen }}: \widehat{\bar{Y}}_{\text {gen }} \rightarrow \mathbb{P}^{1}
$$

be the double cover of the family $\bar{\epsilon}$ branched over two generic fibers. $\widehat{\bar{Y}}_{g e n}$ is related to $\widehat{\bar{Y}}$ by conifold transitions. Indeed, moving the branch points in $\mathbb{P}^{1}$ to 0 and $\infty$ defines a deformation of $\widehat{\bar{Y}}_{g e n}$ to $\widehat{\bar{Y}}_{\text {sing }}$. Let $\mathrm{H}_{2}\left(\widehat{\bar{Y}}_{g e n}, \mathbb{Z}\right)_{f}$ be the subspace of the fiber classes of $\widehat{\bar{Y}}_{g e n}$. We have

$$
H_{2}\left(\widehat{\bar{Y}}_{\text {gen }}, \mathbb{Z}\right)_{f} \cong H_{2}\left(\widehat{\bar{Y}}_{\text {sing }}, \mathbb{Z}\right)_{f} \cong H_{2}(\bar{Y}, \mathbb{Z})_{f},
$$

where the first isomorphism is proven in [26, page 167] and the second isomorphism is from Lemma 11. The Gromov-Witten theory of $\widehat{\bar{Y}}_{\text {gen }}$ and $\widehat{\widehat{Y}}$ are related by a theorem of Li and Ruan [26, Theorem B]. There is a surjective homomorphism

$$
\phi: H_{2}(\widehat{\bar{Y}}, \mathbb{Z})_{f} \rightarrow H_{2}\left(\widehat{\bar{Y}}_{\text {gen }}, \mathbb{Z}\right)_{f},
$$

so that the Gromov-Witten invariants of $\widehat{\bar{Y}}_{\text {gen }}$ and $\widehat{\bar{Y}}$ have the following relation:

$$
N_{\beta}^{g}\left(\widehat{\bar{Y}}_{\text {gen }}\right)=\sum_{\beta^{\prime} \in \phi^{-1}(\beta)} N_{\beta^{\prime}}^{g}(\widehat{\bar{Y}})
$$

for any $\beta \in H_{2}\left(\widehat{\bar{Y}}_{\text {gen }}, \mathbb{Z}\right)_{f}$ with $\beta \neq 0$. Note that by the identifications of the source and target of $\phi$ with respectively $H_{2}(\widehat{Y}, \mathbb{Z})$ and $H_{2}(Y, \mathbb{Z})$ given above, $\phi$ can be identified with the homomorphism

$$
\widehat{\bar{f}}_{*}: H_{2}(\widehat{\bar{Y}}, \mathbb{Z})_{f} \rightarrow H_{2}(\bar{Y}, \mathbb{Z})_{f}
$$

Thus we can write

$$
\sum_{\substack{\beta \in H_{2}(\bar{Y})_{f} \\ \beta \neq 0}} N_{\beta}^{g}\left(\widehat{\bar{Y}}_{g e n}\right) q^{\beta}=\sum_{\substack{\beta^{\prime} \in H_{2}(\hat{\bar{Y}})_{f} \\ \hat{\bar{f}}_{*}\left(\beta^{\prime}\right) \neq 0}} N_{\beta^{\prime}}^{g}(\widehat{\bar{Y}}) q^{\widehat{\bar{f}}_{*}\left(\beta^{\prime}\right)} .
$$

Observing that

$$
\widehat{q}^{\beta} \mapsto q^{\hat{\bar{f}}_{*}(\beta)}
$$


is the same as the specialization (6), we obtain

$$
Z_{f}^{\hat{\bar{Y}}_{\text {gen }}}(q, \lambda)=\frac{Z_{f}^{\hat{\bar{Y}}}(\widehat{q}, \lambda)}{\left.Z_{f}^{\hat{\bar{Y}}}(\widehat{q}, \lambda)\right|_{q=0}}
$$

after the specialization (하). We must divide by the factor $\left.Z_{f}^{\hat{\bar{Y}}}(\widehat{q}, \lambda)\right|_{q=0}$ in order to compensate for the lack of terms with $\widehat{\bar{f}}\left(\beta^{\prime}\right)=0$ in the above sum.

It remains to be shown that

$$
Z_{f}^{\hat{\bar{Y}}_{e n}}(q, \lambda)=Z_{f}^{\bar{Y}}(q, \lambda)^{2} .
$$

The proof of this is very similar to the degeneration argument used by Maulik and Pandharipande for the computations of the fiber class Gromov-Witten theory of Enriques Calabi-Yau threefold [30, §1.1,§1.4] and we thank Davesh Maulik for calling our attention to it.

Since $\widehat{\bar{Y}}_{\text {gen }}$ is given by a double cover of $\bar{Y}$ branched over two smooth fibers, we can obtain a degeneration of $\widehat{\bar{Y}}_{\text {gen }}$ by allowing the two branched fibers to come together. This forms a good degeneration in the sense that the total space of the degeneration is smooth and that the limiting variety is

$$
\bar{Y} \cup_{F} \bar{Y}
$$

the union of two copies of $\bar{Y}$ in a normal crossing along a smooth fiber $F$. This is the setting in which we can apply relative GromovWitten theory to compute the invariants of $\widehat{\bar{Y}}_{g e n}$ in terms of the relative invariants of the pair $(\bar{Y}, F)$. Our situation is particularly amenable to the computation of Gromov-Witten invariants by degeneration for two reasons. One is that we only consider fiber curve classes and they intersect trivially with $F$. The other is that the degeneration locus $F$ is a (non-compact) $K 3$ surface and consequently, it has no non-trivial Gromov-Witten invariants.

For any fiber class $\beta \in H_{2}\left(\widehat{\bar{Y}}_{\text {gen }}, \mathbb{Z}\right)_{f}$, and any non negative integer $g$ the degeneration formula for Gromov-Witten invariants implies that

$$
N_{\beta}^{g}\left(\widehat{\bar{Y}}_{g e n}\right)=2 N_{\beta}^{g}(\bar{Y} / F),
$$

where the right hand side is the corresponding Gromov-Witten invariant of $\bar{Y}$ relative to $F$. However, since $F$ is a (non-compact) $K 3$ surface (see the proof of Lemma 2 in [30])

$$
N_{\beta}^{g}(\bar{Y} / F)=N_{\beta}^{g}(\bar{Y}) .
$$


The last two equalities immediately imply that

$$
Z_{f}^{\hat{\bar{Y}}_{\text {gen }}}(q, \lambda)=Z_{f}^{\bar{Y}}(q, \lambda)^{2}
$$

The proof of Proposition is now complete.

We have now completed the proof of equations (1)-(4) and so the proof of Theorem 1 is complete.

\section{Crepant Resolution Conjecture}

3.1. Overview. A well known principle in physics asserts that string theory on a Calabi-Yau orbifold $X$ is equivalent to string theory on any crepant resolution $Y \rightarrow X$. Consequently, it is expected that Gromov-Witten theory on $Y$ should be equivalent to Gromov-Witten theory on $X$ and this is known as the crepant resolution conjecture. The crepant resolution conjecture goes back to Ruan [37] and has recently undergone successive refinements [8, 17, 18]. In the case where the orbifold satisfies the hard Lefschetz condition [8, Defn 1.1], the crepant resolution conjecture says that the Gromov-Witten potentials of $X$ and $Y$ are equal after a change of variables. In lemma 24 we prove that the orbifold $\left[\mathbb{C}^{3} / G\right]$ satisfies the hard Lefschetz condition if and only if $G$ is a finite subgroup of $S U(2) \subset S U(3)$ or $S O(3) \subset S U(3)$.

In this section we use the crepant resolution conjecture to derive Conjecture 4 and we prove Proposition 5. We will need to work with the full genus zero Gromov-Witten potential of $Y$, which is a generating function for the invariants of $Y$ with an arbitrary number of insertions. Because $Y$ is Calabi-Yau, the n-point Gromov-Witten invariants are easily obtained from the 0-point invariants along with classical intersections. The main work of this section is computing the triple intersections on $Y$ and manipulating the generating functions.

\subsection{Triple intersections on $Y$ and the genus zero potential func-} tion. Let $\left\{\gamma_{\rho}\right\}_{\rho \in \operatorname{Irr}^{*}(G)}$ be the basis for $H^{2}(Y, \mathbb{Q})$ dual to $\left\{C_{\rho}\right\}$, namely that satisfies

$$
\int_{C_{\rho}} \gamma_{\rho^{\prime}}=\delta_{\rho \rho^{\prime}}
$$

Let $L_{\rho}$ be the line bundle on $Y$ defined by $c_{1}\left(L_{\rho}\right)=\gamma_{\rho}$. We choose a lift of the $\mathbb{C}^{*}$-action of $Y$ to $L_{\rho}$ such that

$$
\int_{Y} c_{1}\left(L_{\rho}\right)=0
$$


where the integral is evaluated via localization. We also denote by $\gamma_{\rho}$ the equivariant Chern class of $L_{\rho}$ with the chosen lift. We take $\gamma_{0}$, corresponding to the trivial 1-dimensional representation of $G$, to be the identity in equivariant cohomology. We have that $\left\{\gamma_{\rho}\right\}_{\rho \in \operatorname{Irr}(G)}$ is a set of generators for $H_{\mathbb{C}^{*}}^{*}(Y, \mathbb{Q})$, the equivariant cohomology ring of $Y$. Let $y=\left\{y_{\rho}\right\}_{\rho \in \operatorname{Irr}(G)}$ be a set of variables corresponding to this cohomology basis.

For any given vector $m=\left(m_{\rho}\right)_{\rho \in \operatorname{Irr}(G)}$ of nonnegative integers, we use the following notation

$$
\gamma^{m}=\prod_{\rho \in \operatorname{Irr}(G)} \gamma_{\rho}^{m_{\rho}} \text { and } \frac{y^{m}}{m !}=\prod_{\rho \in \operatorname{Irr}(G)} \frac{y_{\rho}^{m_{\rho}}}{m_{\rho} !} .
$$

Let $\beta \in H_{2}(Y, \mathbb{Z}), g \in \mathbb{Z}_{+}$, and suppose that

$$
|m|=\sum_{\rho \in \operatorname{Irr}(G)} m_{\rho}
$$

The genus $g$, degree $\beta,|m|$-point equivariant Gromov-Witten invariant of $Y$ corresponding to the vector $m$ is denoted by $\left\langle\gamma^{m}\right\rangle_{g, \beta}^{Y}$. In general, these invariants take values in $\mathbb{Q}(t)$ (see [12]). Note that we previously denoted the 0-point invariants, \langle\rangle$_{g, \beta}^{Y}$, by $N_{\beta}^{g}(Y)$.

Definition 16. Using the notation above, we write the genus zero potential function for Gromov-Witten invariants of $Y$ as

$$
F^{Y}(q, y)=\sum_{\beta \in H_{2}(Y, Z)} \sum_{m \in \mathbb{Z}_{+}^{r}}\left\langle\gamma^{m}\right\rangle_{0, \beta}^{Y} q^{\beta} \frac{y^{m}}{m !}
$$

where $r=|\operatorname{Irr}(G)|$. We call the $\beta=0$ part of $F^{Y}$ the classical part, and the $\beta \neq 0$ part of $F^{Y}$ the quantum part. We denote the former by $F_{c l}^{Y}$, and the latter by $F_{q u}^{Y}$.

$F_{c l}^{Y}$ involves only the classical triple equivariant intersections on $Y$. In other words, $F_{c l}^{Y}$ is the cubic polynomial in $y$ given by

$$
F_{c l}^{Y}(y)=\sum_{\rho, \rho^{\prime}, \rho^{\prime \prime} \in \operatorname{Irr}(G)} \int_{Y} \gamma_{\rho} \gamma_{\rho^{\prime}} \gamma_{\rho^{\prime \prime}} \frac{y \rho y \rho^{\prime} y_{\rho^{\prime \prime}}}{3 !} .
$$

If $\beta \neq 0$ then using the point and divisor axioms, one can write all the Gromov-Witten invariants in terms of 0-point invariants \langle\rangle$_{g}^{Y}, \beta$.

Remark 17. One can see that for any $\rho \in \operatorname{Irr}^{*}(G)$, the variables $y_{\rho}$ and $q_{\rho}$ always appear in $F_{q u}^{Y}$ as the product $q_{\rho} e^{y_{\rho}}$. Thus if we set $y=0$ in $F^{Y}$ no information will be lost from its quantum part. 
We determine the classical and the quantum parts of $F^{Y}$ in separate propositions.

Proposition 18. The classical triple equivariant intersection numbers on $Y$ are given by

$$
\begin{aligned}
\int_{Y} \gamma_{0} & =\frac{1}{t^{3}|G|^{\prime}} \\
\int_{Y} \gamma_{\rho} & =0 \\
\int_{Y} \gamma_{\rho} \gamma_{\rho^{\prime}} & =\frac{-1}{2 t h} \sum_{\alpha \in R^{+}} \alpha^{\rho} \alpha^{\rho^{\prime}} \\
\int_{Y} \gamma_{\rho} \gamma_{\rho^{\prime}} \gamma_{\rho^{\prime \prime}} & =\frac{1}{4} \sum_{\alpha \in R^{+}} \alpha^{\rho} \alpha^{\rho^{\prime}} \alpha^{\rho^{\prime \prime}}
\end{aligned}
$$

where, $t$ is the equivariant parameter, $h$ is the Coxeter number of the root system $R$, and $\alpha^{\rho}$ is the coefficient of the simple root corresponding to $\rho$ in the positive root $\alpha$.

PROOF: Recall the map $\widehat{f}: \widehat{Y} \rightarrow Y$ constructed in $\$ 2.2$. For any $\rho \in \operatorname{Irr}^{*}(G)$, the $\mathbb{C}^{*}$-equivariant line bundle $L_{\rho}$ on $Y$ defined above, pulls back via $\widehat{f}$ to a $\mathbb{C}^{*}$-equivariant line bundle on $\widehat{Y}$ denoted by $\widehat{L}_{\widehat{\rho}}$. By the push-pull formula for $\widehat{f}$, we have

$$
\begin{gathered}
\int_{\widehat{C}_{\widehat{\rho}^{\prime}}} c_{1}\left(\widehat{L}_{\widehat{\rho}}\right)=\delta_{\widehat{\rho} \widehat{\rho}^{\prime}} \\
\int_{\widehat{S}} c_{1}\left(\widehat{L}_{\widehat{\rho}}\right)=0
\end{gathered}
$$

for any $\widehat{\rho}, \widehat{\rho}^{\prime} \in \operatorname{Irr}^{*}(G) \subset \operatorname{Irr}^{*}(\widehat{G})$. We extend $\left\{\widehat{L}_{\widehat{\rho}}\right\}_{\widehat{\rho} \in \operatorname{Irr}^{*}(G)}$ to the set of $\mathbb{C}^{*}$-equivariant line bundles $\left\{\widehat{L}_{\hat{\rho}}\right\}_{\widehat{\rho} \in \operatorname{Irr}^{*}(\widehat{G})}$ satisfying the two relations above for any $\widehat{\rho}, \widehat{\rho}^{\prime} \in \operatorname{Irr}^{*}(\widehat{G})$. Define $\widehat{\gamma}_{\hat{\rho}}=c_{1}\left(\widehat{L}_{\widehat{\rho}}\right)$, and let $\widehat{\gamma}_{0}$ be the class of identity in $H_{\mathbb{C}^{*}}^{*}(\widehat{S}, \mathbb{Q})$, the equivariant cohomology of $\widehat{S}$. By construction, $\left\{\widehat{\gamma}_{\hat{\rho}}\right\}_{\widehat{\rho} \in \operatorname{Irr}(\widehat{G})}$ is a basis for $H_{\mathbb{C}^{*}}^{*}(\widehat{S}, \mathbb{Q})$.

Now let $\widehat{\gamma} \in H_{\mathbb{C}^{*}}^{*}(\widehat{Y}, \mathbb{Q})$ be the pull back of some $\gamma \in H_{\mathbb{C}^{*}}^{*}(Y, \mathbb{Q})$ via the $\mathbb{C}^{*}$-equivariant map $\widehat{f}$. We can write

$$
\int_{Y} \gamma=\frac{1}{2} \int_{\widehat{Y}} \widehat{\gamma}=\frac{1}{2 t} \int_{\widehat{S}} \widehat{\gamma}
$$

All the integrals are defined via localization. The factor $1 / 2$ in the first equality appears because $\widehat{f}$ is generically a double cover map, and the $1 / t$ factor in the second equality because the weight of the 
normal bundle of $\widehat{S} \hookrightarrow \widehat{Y}$ is 1 . Proposition 18 follows immediately from these identities and Lemma19.

Lemma 19. Let $\left\{\widehat{\gamma}_{\hat{\rho}}\right\}_{\widehat{\rho} \in \operatorname{Irr}(\widehat{G})}$ be the basis for $H_{\mathbb{C}^{*}}^{*}(\widehat{S}, \mathbb{Q})$ chosen above. Then we have

$$
\begin{aligned}
\int_{\widehat{S}} \widehat{\gamma}_{0} & =\frac{4}{t^{2}|\widehat{G}|}, \\
\int_{\widehat{S}} \widehat{\gamma}_{\widehat{\rho}} & =0, \\
\int_{\widehat{S}} \widehat{\gamma}_{\widehat{\rho}} \widehat{\gamma}_{\widehat{\rho}^{\prime}} & =\frac{-1}{h} \sum_{\alpha \in R^{+}} \alpha^{\widehat{\rho}} \alpha^{\widehat{\rho}^{\prime}}, \\
\int_{\widehat{S}} \widehat{\gamma}_{\widehat{\rho}} \widehat{\gamma}_{\widehat{\rho}^{\prime}} \widehat{\gamma}_{\widehat{\rho}^{\prime \prime}} & =\frac{t}{2} \sum_{\alpha \in R^{+}} \alpha^{\widehat{\rho}} \alpha^{\widehat{\rho}^{\prime}} \alpha^{\widehat{\rho}^{\prime \prime}},
\end{aligned}
$$

PROOF: This is basically [6, Lemma 4] with only the following differences

1. The basis chosen for $H_{\mathrm{C}^{*}}^{*}(\widehat{S}, \mathbb{Q})$ is obtained from the basis chosen in [6] by the negative inverse of the Cartan matrix of $R$. The reason for this is that the cohomology basis chosen in [6] is the dual of $\left\{C_{\widehat{\rho}}\right\}_{\hat{\rho} \in \operatorname{Irr}^{*}(\widehat{G})}$ with respect to the intersection pairing (which is the negative Cartan matrix by the McKay correspondence), while in this paper it is the dual of $\left\{C_{\widehat{\rho}}\right\}_{\widehat{\rho} \in \operatorname{Irr}^{*}(\widehat{G})}$ with respect to the equivariant Poincaré pairing.

2. The weights of the induced $\mathbb{C}^{*}$-action on the canonical bundle of $\widehat{S}$ and the cohomology basis elements $\widehat{\gamma}_{\hat{\rho}}$ are half of the corresponding weights in [6].

Remark 20. Note that (8) in Proposition 18 is the direct result of the definition of $\left\{\gamma_{\rho}\right\}$. (7) in Proposition 18 can also be deduced by the same argument as in the proof of Lemma 4 in [6], by pushing forward the class of the identity to $H_{\mathbb{C}^{*}}^{0}(\mathcal{X})$.

Remark 21. Define the matrix $G=\left(g_{\rho \rho}\right)_{\rho, \rho^{\prime} \in \operatorname{Irr}^{*}(G)}$ by

$$
g_{\rho \rho^{\prime}}=\int_{Y} \gamma_{\rho} \gamma_{\rho^{\prime}}
$$

Then one can show that $G^{-1}=\left(g^{\rho \rho^{\prime}}\right)$ with

$$
g^{\rho \rho^{\prime}}=t\left\langle(V-3 \mathbb{C}) \otimes \rho, \rho^{\prime}\right\rangle
$$


where $\langle\cdot, \cdot\rangle$ is the pairing making the irreducible representations orthonormal. The matrix $G^{-1}$ is the McKay quiver and is the analog of the negative Cartan matrix in dimension 2.

Proposition 22. The genus zero Gromov-Witten potential function of $Y$ is given by

$$
F^{Y}(q, y)=F_{c l}^{Y}(y)+\sum_{\alpha \in R^{+}} \sum_{d=1}^{\infty} \frac{1}{2 d^{3}} q^{d c(\alpha)} e^{d \sum_{\rho} \alpha^{\rho} y_{\rho}} .
$$

PROOF: This is a consequence of Theorem 1 and Remark 17, Each genus zero Gopakumar-Vafa invariant $n_{\beta}^{0}$ contributes $n_{\beta}^{0} d^{-3}$ to the degree $d \beta$ genus zero Gromov-Witten invariant. The proposition follows.

\subsection{Deriving $F^{\mathcal{X}}$ via the crepant resolution conjecture.}

Let $\operatorname{Conj}^{*}(G)$ be the set of nontrivial conjugacy classes of $G$. For $(g) \in \operatorname{Conj}^{*}(G)$ and $\rho \in \operatorname{Irr}^{*}(G)$, we define

$$
L_{\rho}^{(g)}=\frac{|\mathrm{C}(g)|}{|G|} \sqrt{3-\chi_{V}(g)} \chi_{\rho}^{g},
$$

where $\mathrm{C}(g)$ is the centralizer of the group element $g$, and $V$ is the 3 dimensional representation of $G$ induced by the embedding $G \subset$ $S O$ (3). It was conjectured in [8, Conjecture 3.1] that $F^{\mathcal{X}}(x)$ is obtained from $F^{Y}(y, q)$ after replacing

$$
\begin{aligned}
& y_{0}=x_{(e)}, \\
& y_{\rho}=\sum_{(g) \in \operatorname{Conj}^{*}(G)} i L_{\rho}^{(g)} x_{(g)}, \\
& q_{\rho}=\exp \left(\frac{2 \pi i \operatorname{dim}(\rho)}{|G|}\right) .
\end{aligned}
$$

Recalling the definition of $\mathrm{X}_{\rho}$ given in conjecture 4 , we see that

$$
\mathrm{X}_{\rho}=\frac{2 \pi \operatorname{dim}(\rho)}{|G|}+\sum_{(g) \in \text { Conj }^{*}(G)} L_{\rho}^{(g)} x_{(g)} .
$$

Using the above equations to substitute the $y$ variables for the $x$ variables, and specializing the $q$ variables, we obtain the prediction

$$
F^{\mathcal{X}}(x)=F_{c l}^{Y}(x)+\sum_{\alpha \in R^{+}} \frac{1}{2 d^{3}} \exp \left(i d \sum_{\rho} \alpha^{\rho} \mathbf{X}_{\rho}\right) \text {. }
$$


We take the third partial derivatives of the above equation with respect to the variables $x_{(k)}, x_{\left(k^{\prime}\right)}$ and $x_{\left(k^{\prime \prime}\right)}$ corresponding to three nontrivial conjugacy classes, use (4) in Proposition 18, and sum the geometric series to arrive at

$$
\begin{aligned}
& F_{x_{(k)} x_{\left(k^{\prime}\right)} x_{\left(k^{\prime \prime}\right)}}^{\mathcal{X}}(x)=\sum_{\rho, \rho^{\prime}, \rho^{\prime \prime}}\left(\frac{1}{4} \sum_{\alpha \in R^{+}} \alpha^{\rho} \alpha^{\rho^{\prime}} \alpha^{\rho^{\prime \prime}}\right) i^{3} L_{\rho}^{(k)} L_{\rho^{\prime}}^{\left(k^{\prime}\right)} L_{\rho^{\prime \prime}}^{\left(k^{\prime \prime}\right)} \\
& +\sum_{\alpha \in R} \frac{i^{3}}{2}\left(\sum_{\rho} \alpha^{\rho} L_{\rho}^{(k)} \cdot \sum_{\rho^{\prime}} \alpha^{\rho^{\prime}} L_{\rho^{\prime}}^{\left(k^{\prime}\right)} \cdot \sum_{\rho^{\prime \prime}} \alpha^{\rho^{\prime \prime}} L_{\rho^{\prime \prime}}^{\left(k^{\prime \prime}\right)}\right) \frac{e^{i \sum_{\rho} \alpha^{\rho} \mathrm{X}_{\rho}}}{1-e^{i \sum_{\rho} \alpha^{\rho} \mathrm{X}_{\rho}}} .
\end{aligned}
$$

Using the identity

$$
\frac{e^{i \theta}}{1-e^{i \theta}}=-\frac{1}{2}-\frac{i}{2} \tan \left(\frac{\theta}{2}+\frac{\pi}{2}\right),
$$

we find that a delicate cancellation leads to

$$
\begin{gathered}
F_{x_{(k)} x_{\left(k^{\prime}\right)} x_{\left(k^{\prime \prime}\right)}}^{\mathcal{X}}(x)= \\
\frac{-1}{4} \sum_{\alpha \in R^{+}}\left(\sum_{\rho} \alpha^{\rho} L_{\rho}^{(k)} \cdot \sum_{\rho^{\prime}} \alpha^{\rho^{\prime}} L_{\rho^{\prime}}^{\left(k^{\prime}\right)} \cdot \sum_{\rho^{\prime \prime}} \alpha^{\rho^{\prime \prime}} L_{\rho^{\prime \prime}}^{\left(k^{\prime \prime}\right)}\right) \tan \left(\frac{1}{2} \sum_{\rho} \alpha^{\rho} \mathrm{X}_{\rho}+\frac{\pi}{2}\right) .
\end{gathered}
$$

By integrating the above with respect to the variables $x_{(k)}, x_{\left(k^{\prime}\right)}$ and $x_{\left(k^{\prime \prime}\right)}$, we have derived the prediction in Conjecture 4 for $F^{\mathcal{X}}(x)$.

Remark 23. By the point axiom, the only nontrivial terms in $F^{\mathcal{X}}(x)$ containing $x_{(e)}$ are

$$
\left\langle\delta_{(e)}^{3}\right\rangle \frac{x_{(e)}^{3}}{3 !} \quad \text { and } \quad\left\langle\delta_{(e)} \delta_{(g)} \delta_{\left(g^{-1}\right)}\right\rangle x_{(e)} x_{(g)} x_{\left(g^{-1}\right)} \text { for } g \neq e
$$

corresponding to the classical invariants, which are easily evaluated to

$$
\left\langle\delta_{(e)}^{3}\right\rangle=\frac{1}{t^{3}|G|} \quad \text { and } \quad\left\langle\delta_{(e)} \delta_{(g)} \delta_{\left(g^{-1}\right)}\right\rangle=\frac{1}{t|C(g)|} .
$$

By the similar argument given in [6], one can check that these terms match up with the part of $F^{Y}$ with nonzero $y_{0}$ terms (see Remark21).

The above derivation of $F^{\mathcal{X}}(x)$, along with the preceding remark shows that if Conjecture 4 is true, then the Crepant Resolution Conjecture holds for $Y \rightarrow \mathbb{C}^{3} / G$ using the change of variables given by equation (11). This proves Proposition 5 . 


\subsection{The Hard Lefschetz condition.}

Lemma 24. Let $G$ be a finite subgroup of $S U(3)$, then the following conditions are equivalent:

(1) The orbifold $\left[\mathbb{C}^{3} / G\right]$ satisfies the hard Lefschetz condition [8, Defn 1.1].

(2) Every non-trivial element of $G$ has age 1.

(3) $G$ is a finite subgroup of $S O(3) \subset S U(3)$ or $S U(2) \subset S U(3)$.

ProOf: Let $g \in G$ be an element of order $n>1$. The eigenvalues of the action of $g$ on $\mathbb{C}^{3}$ are $\omega^{k_{1}}, \omega^{k_{2}}$, and $\omega^{k_{3}}$, where $\omega=\exp \left(\frac{2 \pi i}{n}\right)$, $k_{i} \in\{0, \ldots, n-1\}$, and $k_{1}+k_{2}+k_{3} \equiv 0 \bmod n$. By definition, the age of the element $g$ is given by

$$
\operatorname{age}(g)=\frac{1}{n}\left(k_{1}+k_{2}+k_{3}\right) \text {. }
$$

By definition, the hard Lefschetz condition means that age $(g)=$ age $\left(g^{-1}\right)$ for all $g$. If $k_{i} \neq 0$ for $i=1,2,3$, then

$$
\operatorname{age}\left(g^{-1}\right)=\frac{1}{n}\left(\left(n-k_{1}\right)+\left(n-k_{2}\right)+\left(n-k_{3}\right)\right)=3-\operatorname{age}(g) .
$$

Consequently, if age $(g)=\operatorname{age}\left(g^{-1}\right)$ then $k_{i}=0$ for some $i$. In this case the eigenvalues must be $\left(1, \omega^{k}, \omega^{n-k}\right)$ and age $(g)=\operatorname{age}\left(g^{-1}\right)=$ 1. This proves that (1) is equivalent to (2).

If $G$ is a subgroup of $S U(2) \subset S U(3)$ or $S O(3) \subset S U(3)$, then every element fixes some line in $\mathbb{C}^{3}$ and hence has age one (or zero). Thus (3) implies (2), and it remains to be shown that (2) implies (3).

Let $\chi_{V}$ be the character of $V$, the 3 dimensional representation induced by $G \subset S U(3)$. By assumption, the action of $g$ on $V$ has eigenvalues $\left\{1, \omega^{k}, \omega^{-k}\right\}$. We have

$$
\begin{aligned}
\chi_{V}\left(g^{2}\right) & =1+\omega^{2 k}+\omega^{-2 k} \\
& =\left(\omega^{k}+\omega^{-k}+1\right)\left(\omega^{k}+\omega^{-k}-1\right) \\
& =\bar{\chi}_{V}(g)\left(\chi_{V}(g)-2\right) .
\end{aligned}
$$

Thus

$$
\frac{1}{|G|} \sum_{g \in G} \chi_{V}\left(g^{2}\right)=\frac{1}{|G|} \sum_{g \in G} \bar{\chi}_{V}(g)\left(\chi_{V}(g)-2\right) .
$$

We decompose $V$ into irreducible summands and we let $r, c$, and $q$ be the number of real, complex, and quaternionic summands respectively and let $t$ be the number of trivial summands. By [21, Ex. 3.38], the left hand side of the above equation is equal to $r-q$ 
while by the orthogonality of characters, the right hand side is equal to $r+c+q-2 t$. Thus we obtain

$$
2 t=c+2 q
$$

which, since $V$ is of dimension 3 , can only be solved by $t=0$ or $t=1$. If $t=0$ then $c=q=0, r=1$, and so $V$ is real, i.e. $G \subset S O(3)$. If $t=1$ then $G \subset S U(2)$ and then lemma is proved.

\section{THE $D_{5}$ CASE: $G=\Sigma_{3}$.}

To illustrate the theorems of this paper, we work out the $D_{5}$ case explicitly. Let $\Sigma_{3} \subset S O(3)$ be the group of permutations of three letters (which is the dihedral group of the triangle and corresponds to $D_{5}$ in the classification). We have

$$
\operatorname{Irr}^{*}\left(\Sigma_{3}\right)=\left\{V_{1}, V_{2}\right\}
$$

where $V_{1}$ and $V_{2}$ are respectively the alternating and the standard representations of $\Sigma_{3}$. The embedding $\Sigma_{3} \subset S O(3)$ induces the 3dimensional representation

$$
V=V_{1} \oplus V_{2} .
$$

The binary version of $\Sigma_{3}$ is the generalized quaternion group of order 12 which we denote by $\widehat{\Sigma}_{3}$. The non-trivial irreducible representations of $\widehat{\Sigma}_{3}$ are

$$
\operatorname{Irr}^{*}\left(\widehat{\Sigma}_{3}\right)=\left\{V_{1}, V_{2}, U_{1}, U_{2}, U_{3}\right\} .
$$

$\widehat{\Sigma}_{3}$ acts on $\mathbb{C}^{2}$ via $U_{1}$. Recall that

$$
\widehat{\pi}: \widehat{S} \rightarrow \mathbb{C}^{2} / \widehat{\Sigma}_{3} \quad \pi: Y \rightarrow \mathbb{C}^{3} / \Sigma_{3}
$$

are the minimal and G-Hilbert scheme resolutions. The intersection graphs of the exceptional sets $\hat{\pi}^{-1}(0)$ and $\pi^{-1}(0)$ are given in Figure 3. The vertices in the graphs are corresponding to the irreducible components of $\hat{\pi}^{-1}(0)$ and $\pi^{-1}(0)$. In each graph two vertices are connected if and only if the corresponding components meet at one point. All the components of $\hat{\pi}^{-1}(0)$ are smooth rational curves having normal bundles isomorphic to $\mathcal{O}_{\mathbb{P}^{1}}(-2)$. The components of $\pi^{-1}(0)$ corresponding to $V_{1}$ and $V_{2}$ are smooth rational curves with normal bundles respectively isomorphic to

$$
\mathcal{O}_{\mathbb{P}^{1}}(-1) \oplus \mathcal{O}_{\mathbb{P}^{1}}(-1) \quad \text { and } \quad \mathcal{O}_{\mathbb{P}^{1}}(1) \oplus \mathcal{O}_{\mathbb{P}^{1}}(-3) .
$$



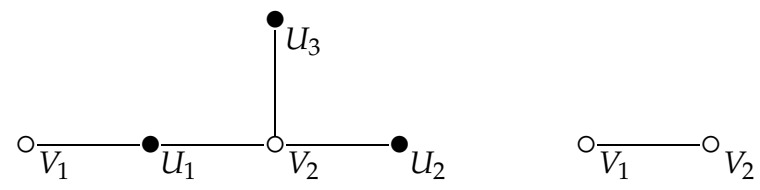

FIGURE 3. The intersection graphs of $\hat{\pi}^{-1}(0)$ and $\pi^{-1}(0)$ respectively.

The morphism $f: \widehat{S} \rightarrow Y$ contracts the components corresponding to the solid vertices. Let $C_{i} \in H_{2}(Y, \mathbb{Z})$ be the curve class corresponding to $V_{i}$ for $i=1,2$. We denote the homology class $d_{1} C_{1}+d_{2} C_{2}$ by $\left(d_{1}, d_{2}\right)$.

Remark 25. In general, the morphism $f: \widehat{S} \rightarrow Y$ contracts disjoint rational curves (see Figure 1). The normal bundle of a curve $C$ in $\pi^{-1}(0)$ is isomorphic to $\mathcal{O}_{\mathbb{P}^{1}}(-k) \oplus \mathcal{O}_{\mathbb{P}^{1}}(k-2)$ where $k$ is the number of components collapsed by $f$ onto $C$. This is a consequence of [32, Theorem 3.2].

The left graph in Figure 3 , is the Dynkin diagram of the $D_{5}$ root system and has vertices in correspondence with the simple roots. There are 20 positive roots in this root system (the first three are the binary roots):

$\begin{array}{cccccccccc}0 & 0 & 1 & 0 & 0 & 0 & 0 & 0 & 1 & 0 \\ 0001 & 0100 & 0000 & 1000 & 0010 & 1100 & 0110 & 0011 & 0010 & 1110 \\ 0 & 1 & 1 & 0 & 1 & 1 & 1 & 1 & 1 & 1 \\ 0111 & 0110 & 0011 & 1111 & 1110 & 0111 & 0121 & 1111 & 1121 & 1221\end{array}$

where the numbers are the coefficients of the corresponding simple root in the graph above.

By Theorem 1, the partition function for the Gromov-Witten invariants of $Y$ is given by

$$
Z^{Y}\left(q_{1}, q_{2}, \lambda\right)=M\left(q_{1}\right) M\left(q_{1} q_{2}\right)^{2} M\left(q_{2}\right)^{4} M\left(q_{2}^{2}\right)^{1 / 2} M\left(q_{1} q_{2}^{2}\right),
$$

where

$$
M(q)=\prod_{m=1}^{\infty}\left(1-q\left(-e^{i \lambda}\right)^{m}\right)^{m} .
$$

The nonzero BPS states are

$$
n_{(1,0)}^{0}=1, \quad n_{(1,1)}^{0}=2, \quad n_{(0,1)}^{0}=4, \quad n_{(0,2)}^{0}=1 / 2, \quad n_{(1,2)}^{0}=1 .
$$

In particular, we obtain a somewhat curious genus zero multiple cover formula for the rational curve $C_{2}$, which has normal bundle 
$\mathcal{O}_{\mathbb{P}^{1}}(1) \oplus \mathcal{O}_{\mathbb{P}^{1}}(-3):$

$$
N_{d C_{2}}^{0}= \begin{cases}\frac{4}{d^{3}} & \text { if } d \text { is odd } \\ \frac{8}{d^{3}} & \text { if } d \text { is even. }\end{cases}
$$

Here the $\mathbb{C}^{*}$ action acts trivially on $C_{2}$ and acts on the normal bundle with weights 2 on the $\mathcal{O}_{\mathbb{P}^{1}}(-3)$ factor and 1 on the $\mathcal{O}_{\mathbb{P}^{1}}(1)$ factor.

Conjecture 4 can be written out explicitly in this case. Let $x_{1}$ and $x_{2}$ be the variables corresponding to the conjugacy class of the transpositions and the three cycles respectively. Then the genus zero orbifold potential function of $\left[\mathbb{C}^{3} / \Sigma_{3}\right]$ is predicted to be

$$
\begin{aligned}
F\left(x_{1}, x_{2}\right)= & \mathbf{h}\left(\frac{4 \pi}{3}-x_{1}+\frac{x_{2}}{\sqrt{3}}\right)+4 \mathbf{h}\left(\frac{\pi}{3}+\frac{x_{2}}{\sqrt{3}}\right)+2 \mathbf{h}\left(x_{1}\right) \\
& +\mathbf{h}\left(\frac{4 \pi}{3}+x_{1}+\frac{x_{2}}{\sqrt{3}}\right)+\frac{1}{2} \mathbf{h}\left(\frac{\pi}{3}-\frac{2 x_{2}}{\sqrt{3}}\right) \\
= & \frac{1}{2} x_{1}^{2} x_{2}+\frac{1}{18} x_{2}^{3}-\frac{5}{48} x_{1}^{4}-\frac{1}{6} x_{1}^{2} x_{2}^{2}-\frac{1}{36} x_{2}^{4}+ \\
& +\frac{1}{12} x_{1}^{4} x_{2}+\frac{1}{18} x_{1}^{2} x_{2}^{3}+\frac{1}{324} x_{2}^{5}+\cdots
\end{aligned}
$$

Since (most of the components of) the moduli space of genus zero maps to $\left[\mathbb{C}^{3} / \Sigma_{3}\right]$ can be identified with the space of degree three admissible covers, the orbifold invariants predicted above can also be viewed as integrals of certain Hodge classes over the moduli space of degree three admissible covers. For example, the special cases where there are exactly two simple ramifications and the rest are double ramification was studied in [9]. The series $B(u)$ which is defined and computed in $[9$, Appendix $\mathrm{A}]$ is given by $F_{112}(0,-u)$ and indeed, the formula

$$
B(u)=\frac{1}{\sqrt{3}} \tan \left(\frac{u}{\sqrt{12}}+\frac{\pi}{3}\right)
$$

given in [9] can be easily recovered from the above prediction for $F\left(x_{1}, x_{2}\right)$.

4.1. Acknowledgements. The authors thank the referees for valuable comments on both technical and expository aspects of this paper. The authors also warmly thank Davesh Maulik, Miles Reid, Rahul Pandharipande, Sophie Terouanne, Michael Thaddeus, and Hsian-Hua Tseng for helpful conversations. The authors also acknowledge support from NSERC, MSRI, the Killam Trust, and the Miller Institute. 


\section{REFERENCES}

[1] Dan Abramovich, Tom Graber, and Angelo Vistoli. Algebraic orbifold quantum products. In Orbifolds in mathematics and physics (Madison, WI, 2001), volume 310 of Contemp. Math., pages 1-24. Amer. Math. Soc., Providence, RI, 2002.

[2] Mina Aganagic, Albrecht Klemm, Marcos Mariño, and Cumrun Vafa. The topological vertex. Comm. Math. Phys., 254(2):425-478, 2005. arXiv:hepth/0305132.

[3] Kai Behrend and Jim Bryan. Super-rigid Donaldson-Thomas invariants. Mathematical Research Letters, 14(4):559-571, 2007. arXiv version: math.AG/0601203.

[4] Samuel Boissiere and Alessandra Sarti. Contraction of excess fibres between the McKay correspondences in dimensions two and three. Ann. Inst. Fourier, 57(6):1839-1861, 2007. arXiv version: math.AG/0504360.

[5] Tom Bridgeland, Alastair King, and Miles Reid. The McKay correspondence as an equivalence of derived categories. J. Amer. Math. Soc., 14(3):535-554 (electronic), 2001.

[6] Jim Bryan and Amin Gholampour. Root systems and the quantum cohomology of ADE resolutions. Algebra and Number Theory, 2(4):369-390, 2008. arXiv:0707.1337.

[7] Jim Bryan and Amin Gholampour. Hurwitz-Hodge integrals, the $E_{6}$ and $D_{4}$ root systems, and the Crepant Resolution Conjecture. Advances in Mathematics, 221(4):1047-1068, 2009. arXiv:0708.4244.

[8] Jim Bryan and Tom Graber. The crepant resolution conjecture. In Algebraic Geometry-Seattle 2005, volume 80 of Proc. Sympos. Pure Math., pages 23-42. Amer. Math. Soc., Providence, RI, 2009. arXiv: math.AG/0610129.

[9] Jim Bryan, Tom Graber, and Rahul Pandharipande. The orbifold quantum cohomology of $\mathbf{C}^{2} / \mathbf{Z}_{3}$ and Hurwitz Hodge integrals. Journal of Algebraic Geometry, 17:1-28, 2008. arXiv version:math.AG/0510335.

[10] Jim Bryan, Sheldon Katz, and Naichung Conan Leung. Multiple covers and the integrality conjecture for rational curves in Calabi-Yau threefolds. J. Algebraic Geom., 10(3):549-568, 2001. Preprint version: math.AG/9911056.

[11] Jim Bryan and Rahul Pandharipande. BPS states of curves in Calabi-Yau 3folds. Geom. Topol., 5:287-318 (electronic), 2001. arXiv: math.AG/0009025.

[12] Jim Bryan and Rahul Pandharipande. The local Gromov-Witten theory of curves. Journal of the American Mathematical Society, 21:101-136, 2008. arXiv:math.AG/0411037.

[13] Renzo Cavalieri. Generating Functions for Hurwitz-Hodge Integrals. math.AG/math/0608590.

[14] Weimin Chen and Yongbin Ruan. Orbifold Gromov-Witten theory. In Orbifolds in mathematics and physics (Madison, WI, 2001), volume 310 of Contemp. Math., pages 25-85. Amer. Math. Soc., Providence, RI, 2002.

[15] Alessandro Chiodo. Towards an enumerative geometry of the moduli space of twisted curves and $r$-th roots. arXiv:math/0607324.

[16] Tom Coates, Alessio Corti, Hiroshi Iritani, and Hsian-Hua Tseng. The Crepant Resolution Conjecture for Type A Surface Singularities. arXiv:0704.2034v1 [math.AG]. 
[17] Tom Coates, Alessio Corti, Hiroshi Iritani, and Hsian-Hua Tseng. Wall-Crossings in Toric Gromov-Witten Theory I: Crepant Examples. arXiv:math.AG/0611550.

[18] Tom Coates and Yongbin Ruan. Quantum Cohomology and Crepant Resolutions: A Conjecture. arXiv:0710.5901.

[19] David A. Cox and Sheldon Katz. Mirror symmetry and algebraic geometry. American Mathematical Society, Providence, RI, 1999.

[20] Alexandru Dimca. Singularities and topology of hypersurfaces. Universitext. Springer-Verlag, New York, 1992.

[21] William Fulton and Joe Harris. Representation theory. Springer-Verlag, New York, 1991. A first course, Readings in Mathematics.

[22] Rajesh Gopakumar and Cumrun Vafa. M-theory and topological strings-II, 1998. Preprint, hep-th/9812127.

[23] Heisuke Hironaka. Triangulations of algebraic sets. In Algebraic geometry (Proc. Sympos. Pure Math., Vol. 29, Humboldt State Univ., Arcata, Calif., 1974), pages 165-185. Amer. Math. Soc., Providence, R.I., 1975.

[24] Kentaro Hori, Sheldon Katz, Albrecht Klemm, Rahul Pandharipande, Richard Thomas, Cumrun Vafa, Ravi Vakil, and Eric Zaslow. Mirror symmetry, volume 1 of Clay Mathematics Monographs. American Mathematical Society, Providence, RI, 2003. With a preface by Vafa.

[25] Sheldon Katz and David R. Morrison. Gorenstein threefold singularities with small resolutions via invariant theory for Weyl groups. J. Algebraic Geom., 1(3):449-530, 1992.

[26] An-Min Li and Yongbin Ruan. Symplectic surgery and Gromov-Witten invariants of Calabi-Yau 3-folds. Invent. Math., 145(1):151-218, 2001.

[27] Jun Li, Chiu-Chu Melissa Liu, Kefeng Liu, and Jian Zhou. A Mathematical Theory of the Topological Vertex. arXiv:math/0408426.

[28] S. Lojasiewicz. Triangulation of semi-analytic sets. Ann. Scuola Norm. Sup. Pisa (3), 18:449-474, 1964.

[29] D. Maulik, N. Nekrasov, A. Okounkov, and R. Pandharipande. GromovWitten theory and Donaldson-Thomas theory. I. Compos. Math., 142(5):12631285, 2006. arXiv:math.AG/0312059.

[30] Davesh Maulik and Rahul Pandharipande. New calculations in GromovWitten theory. arXiv:math.AG/0601395.

[31] John McKay. Graphs, singularities, and finite groups. In The Santa Cruz Conference on Finite Groups (Univ. California, Santa Cruz, Calif., 1979), volume 37 of Proc. Sympos. Pure Math., pages 183-186. Amer. Math. Soc., Providence, R.I., 1980.

[32] David R. Morrison. The birational geometry of surfaces with rational double points. Math. Ann., 271(3):415-438, 1985.

[33] Iku Nakamura. Hilbert schemes of abelian group orbits. J. Algebraic Geom., 10(4):757-779, 2001.

[34] Rahul Pandharipande and Richard Thomas. Curve counting via stable pairs in the derived category. arXiv:math/0707.2348.

[35] Hsian-Hua Tseng Paul Johnson, Rahul Pandharipande. Abelian HurwitzHodge integrals. arXiv:math/0803.0499.

[36] Miles Reid. La correspondance de McKay. Astérisque, (276):53-72, 2002. Séminaire Bourbaki, Vol. 1999/2000. 
[37] Yongbin Ruan. The cohomology ring of crepant resolutions of orbifolds. In Gromov-Witten theory of spin curves and orbifolds, volume 403 of Contemp. Math., pages 117-126. Amer. Math. Soc., Providence, RI, 2006.

[38] Jian Zhou. Crepant resolution conjecture in all genera for type A singularities. arXiv:0811.2023.

[39] Jian Zhou. On computations of Hurwitz-Hodge integrals. arXiv:math/0710.1679.

DEPARTMENT OF MATHEMATICS, UNIVERSITY OF BRITISH COLUMBIA

Department of Mathematics, CAlifornia Institute of TeChNOlogy

E-mail address: jbryan@math.ubc.ca, agholamp@its.caltech.edu 